\title{
Gas Adsorption and Interfacial Tension with Classical Density Functional Theory
}

\author{
Camacho Vergara, Edgar L.; Kontogeorgis, Georgios M.; Liang, Xiaodong
}

Published in:

Industrial \& Engineering Chemistry Research

Link to article, DOI:

10.1021/acs.iecr.9b00137

Publication date:

2019

Document Version

Peer reviewed version

Link back to DTU Orbit

Citation (APA):

Camacho Vergara, E. L., Kontogeorgis, G. M., \& Liang, X. (2019). Gas Adsorption and Interfacial Tension with Classical Density Functional Theory. Industrial \& Engineering Chemistry Research, 58(14), 5650-5664. https://doi.org/10.1021/acs.iecr.9b00137

\section{General rights}

Copyright and moral rights for the publications made accessible in the public portal are retained by the authors and/or other copyright owners and it is a condition of accessing publications that users recognise and abide by the legal requirements associated with these rights.

- Users may download and print one copy of any publication from the public portal for the purpose of private study or research.

- You may not further distribute the material or use it for any profit-making activity or commercial gain

- You may freely distribute the URL identifying the publication in the public portal

If you believe that this document breaches copyright please contact us providing details, and we will remove access to the work immediately and investigate your claim 


\section{Thermodynamics, Transport, and Fluid Mechanics}

Subscriber access provided by DTU Library

\section{Gas adsorption and Interfacial Tension with Classical Density Functional Theory}

Edgar Luis Camacho Vergara, Georgios M. Kontogeorgis, and Xiaodong Liang

Ind. Eng. Chem. Res., Just Accepted Manuscript • DOI: 10.1021/acs.iecr.9b00137 • Publication Date (Web): 11 Mar 2019

Downloaded from http://pubs.acs.org on March 19, 2019

\section{Just Accepted}

"Just Accepted" manuscripts have been peer-reviewed and accepted for publication. They are posted online prior to technical editing, formatting for publication and author proofing. The American Chemical Society provides "Just Accepted" as a service to the research community to expedite the dissemination of scientific material as soon as possible after acceptance. "Just Accepted" manuscripts appear in full in PDF format accompanied by an HTML abstract. "Just Accepted" manuscripts have been fully peer reviewed, but should not be considered the official version of record. They are citable by the Digital Object Identifier (DOI®). "Just Accepted" is an optional service offered to authors. Therefore, the "Just Accepted" Web site may not include all articles that will be published in the journal. After a manuscript is technically edited and formatted, it will be removed from the "Just Accepted" Web site and published as an ASAP article. Note that technical editing may introduce minor changes to the manuscript text and/or graphics which could affect content, and all legal disclaimers and ethical guidelines that apply to the journal pertain. ACS cannot be held responsible for errors or consequences arising from the use of information contained in these "Just Accepted" manuscripts. 
Gas adsorption and Interfacial Tension with Classical Density Functional Theory

\author{
Edgar L. Camacho Vergara, Georgios M. Kontogeorgis, and Xiaodong Liang* \\ Center for Energy Resources Engineering (CERE), Department of Chemical and \\ Biochemical Engineering, Technical University of Denmark, 2800 Kgs. Lyngby, DK \\ E-mail: xlia@kt.dtu.dk \\ Phone: +4545252877
}

\begin{abstract}
A Classical Density Functional Theory (DFT) that reduces to the Perturbed Chain Statistical Associating Fluid Theory equation of state has been implemented. This DFT implementation allows the use of molecular and binary interactions parameters fitted to vapor-liquid equilibrium of pure compounds and binary mixtures, respectively. Different functionals of dispersion forces are investigated and applied to the calculation of interfacial tension and adsorption of pure gases and their mixtures. The predictions of interfacial tension have been compared with both molecular simulations and experimental data, and good agreements have been seen. The DFT approaches also show good performance in correlating and predicting gas adsorption, and they are further compared with Multicomponent Potential Theory of Adsorption and other DFT approaches available in the literature.
\end{abstract}




\section{Introduction}

The study of fluid-fluid, and solid-fluid interfaces is of great interest for the chemical, biochemical and petroleum industries as it is related to a great number of engineering applications, all encompassed in the subject of surface thermodynamics. These applications range from the formation of emulsions and dispersions for food, medicine, detergents, paints and coatings, to improving oil recovery by the addition of surfactants to an oil reservoir, or understanding the process of fluid adsorption on a catalyst pellet in a chemical reactor. ${ }^{1}$ In order to understand how the previous products work, it is important to be able to accurately calculate the interfacial tension between two fluid phases, and the determination of fluid adsorption on solid walls.

One of the most versatile frameworks for the study of interfaces, or inhomogeneous fluids, is Classical Density Functional Theory (DFT). ${ }^{2}$ The framework of DFT was first developed by Hohenberg and Kohn to determine the ground or lowest-energy state properties of the inhomogeneous electron gas. ${ }^{3}$ Later, it was implemented by Ebner ${ }^{4}$ in the study of simple classical fluids, particularly in the vapor-liquid interface of Lennard-Jones fluids. The key point in DFT is the definition of an adequate Helmholtz free energy functional of the particle density of the inhomogeneous system. For the cases of the vapor-liquid and solid-fluid interfaces, the density profiles show uniformities along the axis orthogonal to the interfaces, and are explicitly taken into account by the free energy functional. When it is the case of a bulk system, the density profile has an uniform value and the Helmholtz free energy functional shall be equivalent to the free energy of the bulk system. This characteristic of DFT allows the implementation of equations of state for bulk fluids into the study of inhomogeneous systems in a consistent manner. A thorough explanation of the extension of DFT to classical fluids is given by Evans, ${ }^{5}$ and more recently by Hansen and McDonald. ${ }^{6}$ Moreover, several examples of implementations of DFT in different areas of chemical and biochemical engineering can be found in previous reviews. ${ }^{2,7-9}$

Most of the effort in the development of DFT is dedicated to the definition of the 
Helmholtz free energy functional, focusing in the extension of bulk models to take into account inhomogeneous systems. A relevant example is the work of Tarazona and Evans, ${ }^{10}$ that used an average particle density together with the Carnahan-Starling (CS) equation of state for hard spheres. ${ }^{11}$ They were able to predict adsorption of a hard sphere gas over a solid wall that was in qualitatively agreement with Monte Carlo simulations. Their approach includes the introduction of a weighting function used to calculate the average density that takes into account the inhomogeneities of the system close to the solid wall. It is defined in such a way that when the systems reaches the bulk limit, the weighted density is equal to the bulk density. This approach is formally known as the Weighted Density Approximation (WDA), ${ }^{12,13}$ and it has been proven to be a successful technique to obtain the properties of the inhomogeneous fluid from bulk systems models.

Currently, in the vast majority of the most recent investigations about classical DFT the Helmholtz free energy functionals are developed by means of the Thermodynamic Perturbation Theory (TPT) of Wertheim, ${ }^{14-17}$ extensively reviewed by Zmpitas and Gross. ${ }^{18}$ Similar to the Statistical Association Fluid Theory (SAFT) equation of state ${ }^{19-21}$ and its variants, the TPT scheme allows the separation of the Helmholtz free energy functional into individual terms, each referring to specific particle interactions. The reference state is usually the hard sphere fluid, followed by chain formation, dispersive, association, polar, electrostatic interactions, among others. As mentioned before, in the bulk limit where the local particle density tends to the bulk density the DFT implementations reduce to the bulk equation of state used to define the free energy functional. The most relevant examples of these implementations are SAFT with potential of variable range (SAFT-VR), ${ }^{22-24}$ interfacial SAFT (iSAFT), ${ }^{25-28}$ the perturbed-chain SAFT (PC-SAFT), ${ }^{29-37}$ the most recently SAFT- $\gamma,{ }^{38-40}$ and the work of Wu et al. ${ }^{41-45}$ In general, all implementations are in good agreement with molecular simulations of hard spheres fluids thanks to the very successful Fundamental Measure Theory (FMT) originally from Rosenfeld, ${ }^{46}$ and later modified simultaneously by Yu and $\mathrm{Wu}(\mathrm{mFMT}),{ }^{41}$ and Roth et. al, (White-Bear FMT). ${ }^{47}$ The contribution due to chain 
formation is usually computed according to Tripathi and Chapman, ${ }^{25}$ or with the term derived by $\mathrm{Yu}$ and $\mathrm{Wu}^{42}$ that makes use of weighted densities defined in mFMT. For associating fluids, the expression used is the one developed by Bymaster and Chapman ${ }^{28}$ and alternatively the one developed by $\mathrm{Yu}$ and $\mathrm{Wu}^{43}$ also based on mFMT. What really differentiates these different approaches is how they define the dispersion contributions to the free energy functionals. Over the last decade most of dispersion contributions were based on the Mean Field Approximation (MFA) ${ }^{48}$ which assumes the radial distribution of function of the inhomogeneous fluid with a pairwise additive potential as unity, ${ }^{23,25-28,49}$ or approximating it to that of the bulk fluid. ${ }^{29,33}$ Other approaches include the work of Gloor et al., ${ }^{22}$ Ghobadi and Elliott, ${ }^{40}$ and the implementation of the quadratic density expansion ${ }^{44}$ and Mean Spherical Approximation (MSA) by Fu and Wu. ${ }^{45,50}$ There have been DFT implementations that use WDA together with the reduced Helmholtz free energy expression of the PC-SAFT equation of state, in particular the work of Shen et al. ${ }^{30-32}$ They calculate the weighted density as a density average over the volume of a sphere with a radius equal to $\lambda d$, where $d$ is the temperature-dependent diameter defined in PC-SAFT and $\lambda$ is a factor related to the width of the potential. ${ }^{51}$ Later, Sauer and Gross ${ }^{36}$ adopted the previously mentioned approach and treated $\lambda$ as an adjustable parameter and showed that it can be treated as a unique parameter for all compounds they studied. They proposed two different dispersion functionals based on WDA, one of which slightly different than the one proposed by Shen et al. ${ }^{31}$ More recently, Wang et al. ${ }^{37}$ proposed a functional also using the PC-SAFT dispersion term in the functional form, and treat it locally together with the weighted densities defined in mFMT in order to account for short range interactions. The long range interactions are included in a non-local functional. Their implementation does not require adjustable parameters and it is able to predict interfacial tension successfully but with slightly higher deviations.

Even though DFT has proven to be an adequate framework for the study of inhomogeneous fluids, and takes shorter computation times than molecular simulations, it still requires higher computational effort and complexity than other models used for the calculation of 


\section{THEORETICAL MODEL}

\section{Classical Density Functional Theory}

The DFT framework is derived in the grand canonical ensemble for a fixed volume $V$, temperature $T$ and chemical potential $\mu$. The particle density of each component varies along a three-dimensional coordinate system $\rho_{i}(\mathbf{r})$. Within the DFT formalism, the grand potential 
functional for a mixture of $n c$-components is given by

$$
\Omega[\boldsymbol{\rho}]=\mathcal{F}[\boldsymbol{\rho}]+\sum_{i}^{\mathrm{nc}} \int \rho_{i}(\mathbf{r})\left(\phi_{i}(\mathbf{r})-\mu_{\mathrm{b}, i}\right) d \mathbf{r}
$$

Where $\phi_{i}(\mathbf{r})$ is the external potential characteristic of the solid-fluid interactions for each component, and $\mathcal{F}[\boldsymbol{\rho}]$ is the intrinsic Helmholtz free energy functional of the particle density of all components $\boldsymbol{\rho}=\left\{\rho_{1}(\mathbf{r}), \ldots, \rho_{\mathrm{nc}}(\mathbf{r})\right\}$, and $\mathbf{r}$ represents the coordinate system.

The equilibrium condition that gives the equilibrium particle density $\left(\boldsymbol{\rho}^{*}\right)$ of the inhomogeneous system under the effect of an external potential is found by minimizing the grand potential according to the inequality $\Omega[\boldsymbol{\rho}] \geq \Omega\left[\boldsymbol{\rho}^{*}\right]$. This implies that the functional derivative of $\Omega[\boldsymbol{\rho}]$ with respect to the particle density of each component equals zero. Therefore, from equation Eq. 1 we get that every point of the inhomogeneous systems is in equilibrium with the bulk fluid,

$$
\mu_{\mathrm{b}, i}=\left.\frac{\delta \mathcal{F}[\boldsymbol{\rho}]}{\delta \rho_{i}(\mathbf{r})}\right|_{\boldsymbol{\rho}=\boldsymbol{\rho}^{*}}+\phi_{i}(\mathbf{r})
$$

The free energy functional can be separated into an ideal and a residual part

$$
\mathcal{F}[\rho]=\mathcal{F}^{\mathrm{id}}[\rho]+\mathcal{F}^{\mathrm{res}}[\rho]
$$

The ideal free energy functional is known exactly, and for convenience it is defined as a density functional in terms of the ideal free energy density $f_{i}^{\text {id }}=\rho_{i}(\mathbf{r})\left(\ln \left(\rho_{i}(\mathbf{r})\right)-1\right)$,

$$
\beta \mathcal{F}^{\mathrm{id}}[\boldsymbol{\rho}]=\sum_{i}^{\mathrm{nc}} \int \rho_{i}(\mathbf{r})\left(\ln \left(\rho_{i}(\mathbf{r})\right)-1\right) d \mathbf{r}
$$

where $\beta=\left(k_{B} T\right)^{-1}$, and $k_{B}$ is the Boltzmann constant. The functional derivative of $\mathcal{F}^{\mathrm{id}}[\boldsymbol{\rho}]$ gives

$$
\beta \frac{\delta \mathcal{F}^{\mathrm{id}}[\rho]}{\delta \rho_{i}(\mathbf{r})}=\ln \rho_{i}(\mathbf{r})
$$


That together with equation Eq. 2 we get an expression for the particle density that must be solved iteratively,

$$
\rho_{i}(\mathbf{r})=\exp \left(\beta \mu_{\mathrm{b}, i}-\beta \frac{\delta \mathcal{F}^{\mathrm{res}}[\boldsymbol{\rho}]}{\delta \rho_{i}(\mathbf{r})}-\beta \phi_{i}(\mathbf{r})\right)
$$

Equation Eq. 6 shows how the inhomogeneities, or deviations from the bulk system, are introduced by the Helmholtz free energy functional and the external potential inside the exponential. The chemical potential of the bulk fluid $\beta \mu_{\mathrm{b}, i}$ is simply calculated with the bulk equation of state. Alternatively, the bulk chemical potential can be calculated with the expression of the free energy functional using a constant value for the density as an input. In this way it can be assured that the implementation is consistent both in the inhomogeneous and bulk systems and no oscillations are introduced by the integration errors used in the computation of the functional.

\section{Residual Helmholtz Free Energy Functional}

The DFT implementation developed in this work is intended to be consistent with PCSAFT, ${ }^{59}$ which for non-associating fluids takes three pure component parameters, the segment diameter $\sigma_{i}$, the segment number $m_{i}$ and the energy parameter $\varepsilon_{i}$. Therefore, the residual Helmholtz free energy functional can be built following PC-SAFT as

$$
\mathcal{F}^{\mathrm{res}}[\boldsymbol{\rho}]=\mathcal{F}^{\mathrm{hs}}[\boldsymbol{\rho}]+\mathcal{F}^{\mathrm{hc}}[\boldsymbol{\rho}]+\mathcal{F}^{\mathrm{disp}}[\boldsymbol{\rho}]
$$

Where $h s, h c$ and disp correspond to the hard-sphere, chain formation and dispersive contributions to the free energy. Moreover, the model takes into account an effective temperaturedependent hard sphere diameter $d_{i}(T)=\sigma_{i}\left(1-0.12 \exp \left(-3 \beta \varepsilon_{i}\right)\right)$. For mixtures, mixing rules are required for the size and energy parameters. The usual Berthelot-Lorentz combining rules are used here, 


$$
\begin{gathered}
\sigma_{i j}=0.5\left(\sigma_{i}+\sigma_{j}\right) \\
\varepsilon_{i j}=\sqrt{\varepsilon_{i} \varepsilon_{j}}\left(1-k_{i j}\right)
\end{gathered}
$$

With a binary interaction parameter $k_{i j}$ that is fitted to vapor-liquid equilibrium (VLE) data.

\section{Hard Sphere Contribution}

The hard sphere functional accounting for volume exclusion is determined according to mFMT, ${ }^{41,47}$ which is based in the bulk equation of state for hard spheres mixtures developed in parallel by Boublík ${ }^{60}$ and Mansoori-Carnahan-Starling-Leeland (BMCSL). ${ }^{61}$ The theory defines the free energy density of the hard sphere as a function of several weighted densities that take into account the 'fundamental measures' of the hard spheres. The free energy functional of the hard sphere fluid in mFMT is expressed for a flat interface as

$$
\beta \mathcal{F}^{\mathrm{hs}}[\boldsymbol{\rho}]=\int f^{\mathrm{hs}}\left(\left\{n_{\alpha}(\mathbf{r})\right\}\right) d \mathbf{r}
$$

Where $f^{\text {hs }}\left(\left\{n_{\alpha}\right\}\right)$ is the free energy density that is only a function of a series of six weighted densities denoted by $n_{\alpha}$, with the general form

$$
n_{\alpha}(\mathbf{r})=\sum_{i=1}^{\mathrm{nc}} m_{i} \int \rho_{i}\left(\mathbf{r}^{\prime}\right) \omega_{i}^{(\alpha)}\left(\left|\mathbf{r}-\mathbf{r}^{\prime}\right|\right) d \mathbf{r}^{\prime}
$$

The weighting functions $\omega_{i}^{(\alpha)}$, and further description of mFMT can be found in references 41,62 . 


\section{Chain Formation Contribution}

The Helmholtz free energy functional related to chain formation used in this work was developed by Tripathi and Chapman. ${ }^{25}$ To form this functional it is assumed that a linear chain-like molecule $i$ is formed by $m$ segments. PC-SAFT works with the molecular densities instead of the segment density as defined in the original functional, which requires a correction as shown by Sauer and Gross. ${ }^{36}$ The resulting expression of the chain functional is

$$
\begin{aligned}
\beta \mathcal{F}^{\mathrm{hc}}[\boldsymbol{\rho}]= & \sum_{i}^{\mathrm{nc}}\left(m_{i}-1\right) \int \rho_{i}(\mathbf{r})\left(\ln \rho_{i}(\mathbf{r})-1\right) d \mathbf{r} \\
& -\sum_{i}^{\mathrm{nc}}\left(m_{i}-1\right) \int \rho_{i}(\mathbf{r})\left(\ln \left(y_{\mathrm{ii}}^{\mathrm{dd}}\left(\bar{\rho}^{\mathrm{hc}}(\mathbf{r})\right) \lambda_{i}(\mathbf{r})\right)-1\right) d \mathbf{r}
\end{aligned}
$$

However, this correction is only required because of the selection of the ideal free energy functional in terms of the molecular density. As shown by Shen et. al, ${ }^{30}$ the original chain functional from Tripathi and Chapman can be used only if the ideal free energy functional is selected accordingly. In equation Eq. 12, $y_{\mathrm{ii}}^{\mathrm{dd}}\left(\bar{\rho}^{\mathrm{hc}}(\mathbf{r})\right)$ is the cavity correlation function between two segments evaluated at contact distance and at an WDA-type averaged density $\bar{\rho}^{\text {hc }}(\mathbf{r})$ corresponding to the interpenetration volume of two segments

$$
\bar{\rho}_{i}^{\mathrm{hc}}(\mathbf{r})=\frac{3}{4 \pi d_{i}^{3}} \int \rho_{i}\left(\mathbf{r}^{\prime}\right) \Theta\left(d_{i}-\left|\mathbf{r}-\mathbf{r}^{\prime}\right|\right) d \mathbf{r}^{\prime}
$$

Also appearing in equation Eq. 12 is an averaged density over a segment surface at contact distance to account for segment-segment connectivity

$$
\lambda_{i}(\mathbf{r})=\frac{1}{4 \pi d_{i}^{2}} \int \rho_{i}\left(\mathbf{r}^{\prime}\right) \delta\left(d_{i}-\left|\mathbf{r}-\mathbf{r}^{\prime}\right|\right) d \mathbf{r}^{\prime}
$$

For this particular case $\delta$ represents the Dirac delta function. 


\section{Dispersive Contribution}

For the dispersive interactions, the free energy functional is approximated directly from the PC-SAFT expression for bulk fluids ${ }^{59}$ by means of an weighted density $\bar{\rho}(\mathbf{r})$ in the form of WDA. ${ }^{10}$ The weighted density can be written as

$$
\bar{\rho}_{i}(\mathbf{r})=\frac{3}{4 \pi\left(\lambda d_{i}\right)^{3}} \int \rho_{i}\left(\mathbf{r}^{\prime}\right) \Theta\left(\lambda d_{i}-\left|\mathbf{r}-\mathbf{r}^{\prime}\right|\right) d \mathbf{r}^{\prime}
$$

Previous implementations of functionals of this form based on the PC-SAFT equations of state have used this approach with a fixed value of $\lambda=1.5 \cdot{ }^{30,31,51}$ This particular value of $\lambda$ allows the accurate calculation of density profiles and adsorption of confined fluids, however, it gives poor predictions for interfacial tension calculations. Later, Sauer and Gross ${ }^{36}$ treated $\lambda$ as an adjustable model parameter that is determined by fitting experimental data of interfacial tension of n-alkanes. This procedure is similar in nature with the universal constants of the dispersion term of the PC-SAFT equation of state.

In this work we use three different approaches to build the functionals, two already introduced by Sauer and Gross, ${ }^{36}$ and extend the one introduced by Shen et al. ${ }^{31}$ with the adjustable $\lambda$ to be able to produce accurate results for interfacial tension. All approaches use the reduced Helmholtz free energy expression for the dispersive contribution of PCSAFT equation of state. The difference between the approaches is how they make use of the weighted density in the functional.

The first approach is a full 'non-local' functional (WDA1 in Ref. 36) defined as

$$
\beta \mathcal{F}^{\mathrm{disp}, \mathrm{D} 1}[\rho]=\int \sum_{i} \sum_{j} \bar{\rho}_{i}(\mathbf{r}) \bar{\rho}_{j}(\mathbf{r}) \Phi_{i j}^{\mathrm{disp}}(\bar{\rho}(\mathbf{r})) d \mathbf{r}
$$

The remaining two are considered 'local' approaches, one given by ${ }^{31}$

$$
\beta \mathcal{F}^{\mathrm{disp}, \mathrm{D} 2}[\rho]=\int \sum_{i} \sum_{j} \rho_{i}(\mathbf{r}) \bar{\rho}_{j}(\mathbf{r}) \Phi_{i j}^{\mathrm{disp}}(\bar{\rho}(\mathbf{r})) d \mathbf{r}
$$


And finally (WDA2 in Ref. 36),

$$
\beta \mathcal{F}^{\mathrm{disp}, \mathrm{D} 3}[\rho]=\int \frac{\rho(\mathbf{r})}{\bar{\rho}(\mathbf{r})} \sum_{i} \sum_{j} \bar{\rho}_{i}(\mathbf{r}) \bar{\rho}_{j}(\mathbf{r}) \Phi_{i j}^{\mathrm{disp}}(\bar{\rho}(\mathbf{r})) d \mathbf{r}
$$

Where

$$
\begin{aligned}
\Phi_{i j}^{\mathrm{disp}}(\bar{\rho}(\mathbf{r}))= & -2 \pi m_{i} m_{j} I_{1}(\bar{\eta}(\mathbf{r}), \bar{m}) \frac{\varepsilon_{i j}}{k_{B} T} \sigma_{i j}^{3} \\
& -\pi m_{i} m_{j} \bar{m} C_{1}(\bar{\eta}(\mathbf{r}), \bar{m}) I_{2}(\bar{\eta}(\mathbf{r}), \bar{m})\left(\frac{\varepsilon_{i j}}{k_{B} T}\right)^{2} \sigma_{i j}^{3}
\end{aligned}
$$

The total weighted density $\bar{\rho}(\mathbf{r})$, weighted segment number $\bar{m}$ and averaged packing fraction $\bar{\eta}$ are given by

$$
\begin{gathered}
\bar{\rho}(\mathbf{r})=\sum_{i} \bar{\rho}_{i}(\mathbf{r}) \\
\bar{m}=\frac{1}{\bar{\rho}(\boldsymbol{r})} \sum_{i} m_{i} \bar{\rho}_{i}(\boldsymbol{r}) \\
\bar{\eta}=\frac{\pi}{6} \sum_{i} m_{i} \bar{\rho}_{i}(\mathbf{r}) d_{i}^{3}
\end{gathered}
$$

The expressions for $C_{1}(\bar{\eta}(\mathbf{r}), \bar{m}), I_{1}(\bar{\eta}(\mathbf{r}), \bar{m})$ and $I_{2}(\bar{\eta}(\mathbf{r}), \bar{m})$ can be found elsewhere. ${ }^{31,36,59}$

\section{Results}

\section{Computational aspects}

The most suitable way to carry out the calculations of interfacial tension and adsorption in slit-like pores is by assuming a planar geometry for the interfaces, where the density varies only over the $z$-coordinate orthogonal to the interface or walls of the pore $\rho_{i}(z)$. This simplification implies that the functionals will become intensive properties with respect to 
the area of the interface or surface.

For interfacial tension there is no external potential and equation Eq. 6 becomes

$$
\rho_{i}(z)=\exp \left(\beta \mu_{\mathrm{b}, i}-\beta \frac{\delta \mathcal{F}^{\mathrm{res}}[\rho]}{\delta \rho_{i}(z)}\right)
$$

In terms of the grand potential functional, the interfacial tension reads

$$
\gamma=\Omega[\rho(z)]+P \int_{0}^{H} d z
$$

Where $\Omega[\rho(z)]$ is the grand potential functional per unit area calculated directly from the DFT implementation and $H$ is the size of the interface. Then

$$
\Omega[\rho(z)]=\int f[\rho(z)] d z-\sum_{i}^{\mathrm{nc}} \mu_{\mathrm{b}, i} \int \rho_{i}(z) d z
$$

with $f[\rho(z)]$ as the free energy density.

For confined fluids, the external potential depends only on the separation between the walls $0 \leq z \leq H$, with $z$ as the length coordinate and $H$ the width of the pore. The external potential describes the interactions between the particles of the fluid and the solid walls. For a fluid between two planar walls, the external potential can be defined as

$$
\phi_{i}(z)= \begin{cases}\phi_{\mathrm{sf}, \mathrm{i}}(z), & 0 \leq z \leq H \\ \infty, & \text { otherwise }\end{cases}
$$

Where $\phi_{\mathrm{sf}, \mathrm{i}}(z)$ is the potential energy between the particles of the walls and the particles of the component $i$ in the mixture. To model these interactions we use the Steele fluid-wall potential $^{63}$

$$
\phi_{s f, i}(z)=2 \pi \rho_{s} \varepsilon_{s f, i} \sigma_{s f, i}^{2} \Delta\left[0.4\left(\frac{\sigma_{s f, i}}{z}\right)^{10}-\left(\frac{\sigma_{s f, i}}{z}\right)^{4}-\frac{\sigma_{s f, i}^{4}}{3 \Delta(z+0.61 \Delta)^{3}}\right]
$$

Where $\rho_{s}=0.114 \AA^{-3}$ is the number particle density of the solid and $\Delta=3.35 \AA$ is 

2

the distance between layers of particles in the solid. The solid-fluid size parameter $\sigma_{s f, i}$ is calculated with the mixing rule shown in equation Eq. 8 with $\sigma_{s}=3.34 \AA$, and the energy parameter $\varepsilon_{s f, i}$ is treated as an adjustable parameter unique for each pair solid-component in the pore system. The excess adsorption of component $i$ confined between two planar walls can be calculated from

$$
\Gamma_{i}=\frac{A^{*}}{2} \int_{\sigma_{\mathrm{sf}, \mathrm{i}}}^{H-\sigma_{\mathrm{sf}, \mathrm{i}}}\left(\rho_{i}(z)-\rho_{\mathrm{b}, i}\right) d z
$$

$A^{*}$ is the specific area of the solid, $\rho_{\mathrm{b}, i}$ is the density of component $i$ in the bulk fluid and the integration limits correspond to the volume accessible to the particles inside the slit-like pore.

The integration of the functionals is carried using the trapezoidal rule with a grid spacing of $\Delta z=0.01 \sigma$, to reduce to a minimum the error introduced by the numerical method. The size of the grid $H$ is selected depending on the application; for interfacial tension calculations is intuitively fixed to $H=100 \sigma_{\max }$. For adsorption, $H$ is considered to be the average pore size, and fitted to experimental data of adsorption of pure compounds.

The minimization of the grand potential is achieved by solving equation Eq. 6 with a damped fixed-point iterative procedure. Each update of the density profile for each component is calculated by mixing the current result $\rho_{i}^{k}$ with a new value obtained with equation Eq. 6 by a factor $\kappa$ according to

$$
\rho_{i}^{k+1}=\rho_{i}^{k}+\kappa\left(\rho_{i}^{\text {new }}-\rho_{i}^{k}\right)
$$

The value of the damping factor is held constant thorough the iterative procedure, and ranges from values of $0.001-0.01$.

The initialization is different for interfacial tension and adsorption. For the latter, the density of each component along the grid is set to be equal to its bulk counterpart, and once the external potential is defined with equation Eq. 26 the calculation starts. For interfacial 
tension the vapor-liquid equilibrium points are calculated first, and then the initial density profile is estimated as ${ }^{29}$

$$
\rho_{i}^{0}(z)=\frac{1}{2}\left(\rho_{b, i}^{L}-\rho_{b, i}^{V}\right) \tanh \left(2.7 \frac{z-0.5 H}{\sigma_{i}}\right)+\frac{1}{2}\left(\rho_{b, i}^{L}+\rho_{b, i}^{V}\right)
$$

Where the indexes 'b', 'L' and 'V' account for bulk, liquid phase and vapor phase, respectively.

In all the following calculations, the pure component parameters used in our DFT implementations are taken from the molecular parameters of the PC-SAFT equations of state as reported by Gross and Sadowski. ${ }^{59}$ The binary interactions parameters $k_{i j}$ used are taken from literature or calculated in this work, all of them obtained by fitting experimental binary VLE data of the system under study.

\section{Interfacial tension}

The implementation of the different approaches of the dispersion contribution to the Helmholtz free energy functional with the 'original' value of the factor $\lambda=1.5^{30}$ does not lead to good experimental agreement in the calculation of interfacial tension. Therefore, it is recommended to treat the factor $\lambda$ as an adjustable parameter. ${ }^{36}$ From here on, the approaches to the dispersive contribution will be labeled DFT-D1, DFT-D2 and DFT-D3 according to equations 16, 17 and 18, respectively.

The fitted values of $\lambda$ to interfacial tension data for each approach are $\lambda_{\mathrm{D} 1}=1.3862$ and $\lambda_{\mathrm{D} 2}=1.8738 .{ }^{36}$ It is worth mentioning that for pure components, DFT-D2 and DFTD3 are equivalent and they share the same value for $\lambda$. The difference between the values of $\lambda$ is not surprising, as discussed by Tarazona and Evans, ${ }^{10}$ the use of WDA serves the purpose of smoothing the density profile in comparison with the local density approximation (LDA). LDA simply inputs the local density of a point in the z-coordinate into the bulk equation of state, similar to DGT. However, it does not produce accurate density profiles as 
WDA, especially at high packing fractions of confined fluids. Therefore, the local approach (D2) requires a larger weighting volume to account for larger oscillations introduced by the local density used in the free energy functional. The calculated interfacial tension of several compounds can be seen in Fig. 1, where the results with both approaches (DFT-D1 and -D2) are virtually identical and agree with the experimental data. DFT-D2 shows slightly higher deviations at the lowest temperatures for each of the compounds investigated.

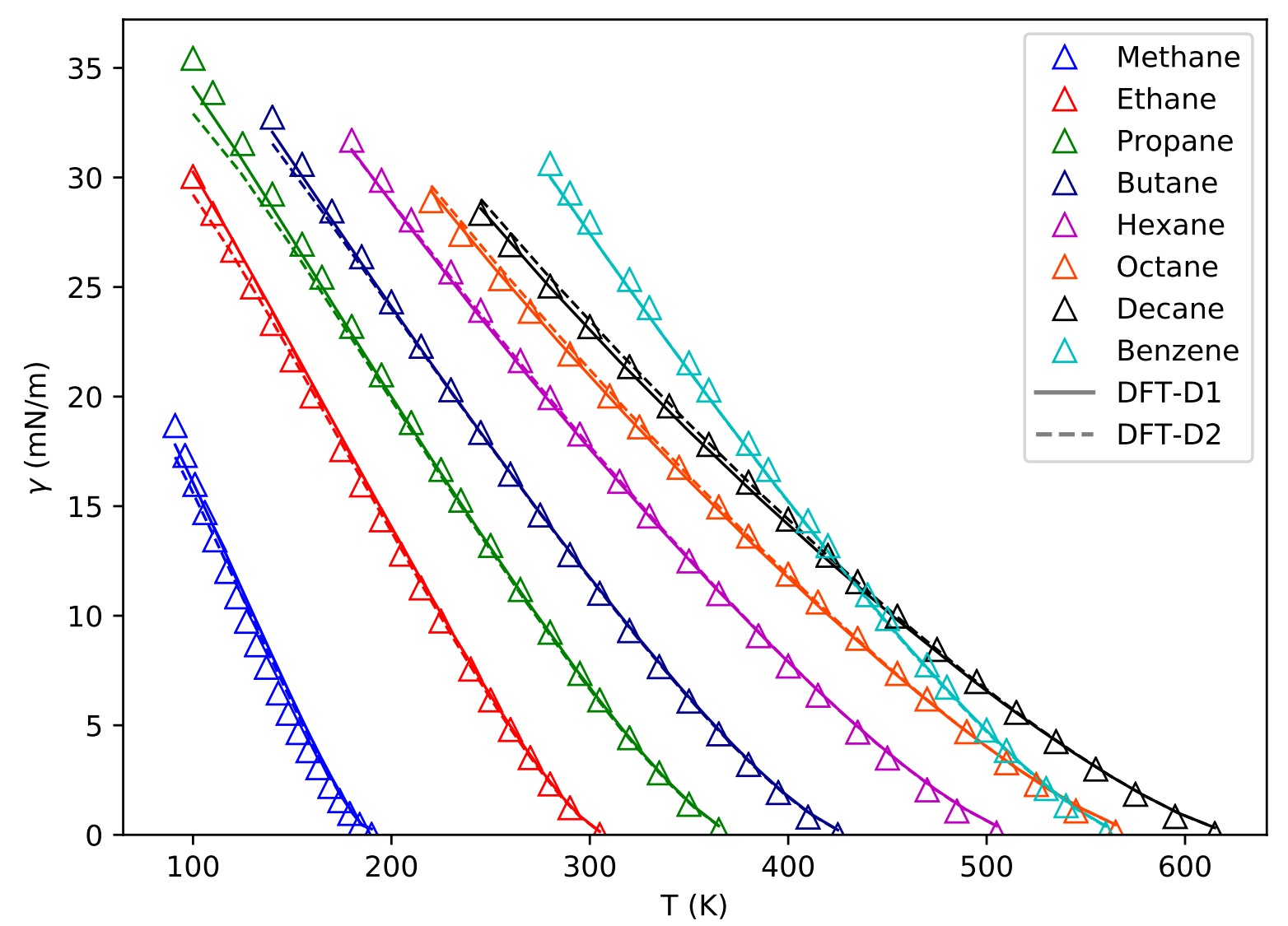

Figure 1: Interfacial tension calculated with DFT-D1, $\lambda=1.3862$ (solid) and DFT-D2, $\lambda=1.8738$ (dashed) for different compounds. ${ }^{64}$

Fig. 2 shows the individual isotherm of the interfacial tension of n-nonane calculated with both DFT-D1 and DFT-D2, and compared to experimental data ${ }^{64}$ and molecular dynamics. ${ }^{65}$ The agreement with experimental data is excellent for both approaches, similar to the performance obtained for the previous compounds. Moreover, DFT shows to be better at 
describing the interfacial tension than molecular dynamics in the temperature range shown in the plot. In addition, Fig. 3 displays the density profiles of the vapor-liquid interface of nnonate at $393 \mathrm{~K}, 435 \mathrm{~K}$ and $475 \mathrm{~K}$ obtained with DFT and compared to molecular dynamics from the same reference. The results from the simulation and DFT give accurate results for the vapor and liquid densities, but the density profiles show some differences. The later may be attributted to the larger deviations of the simulations in the calculation of the interfacial tension. Both DFT approaches give identical density profiles for these temperatures as the calculated interfacial tension are almost identical (see Fig. 2).

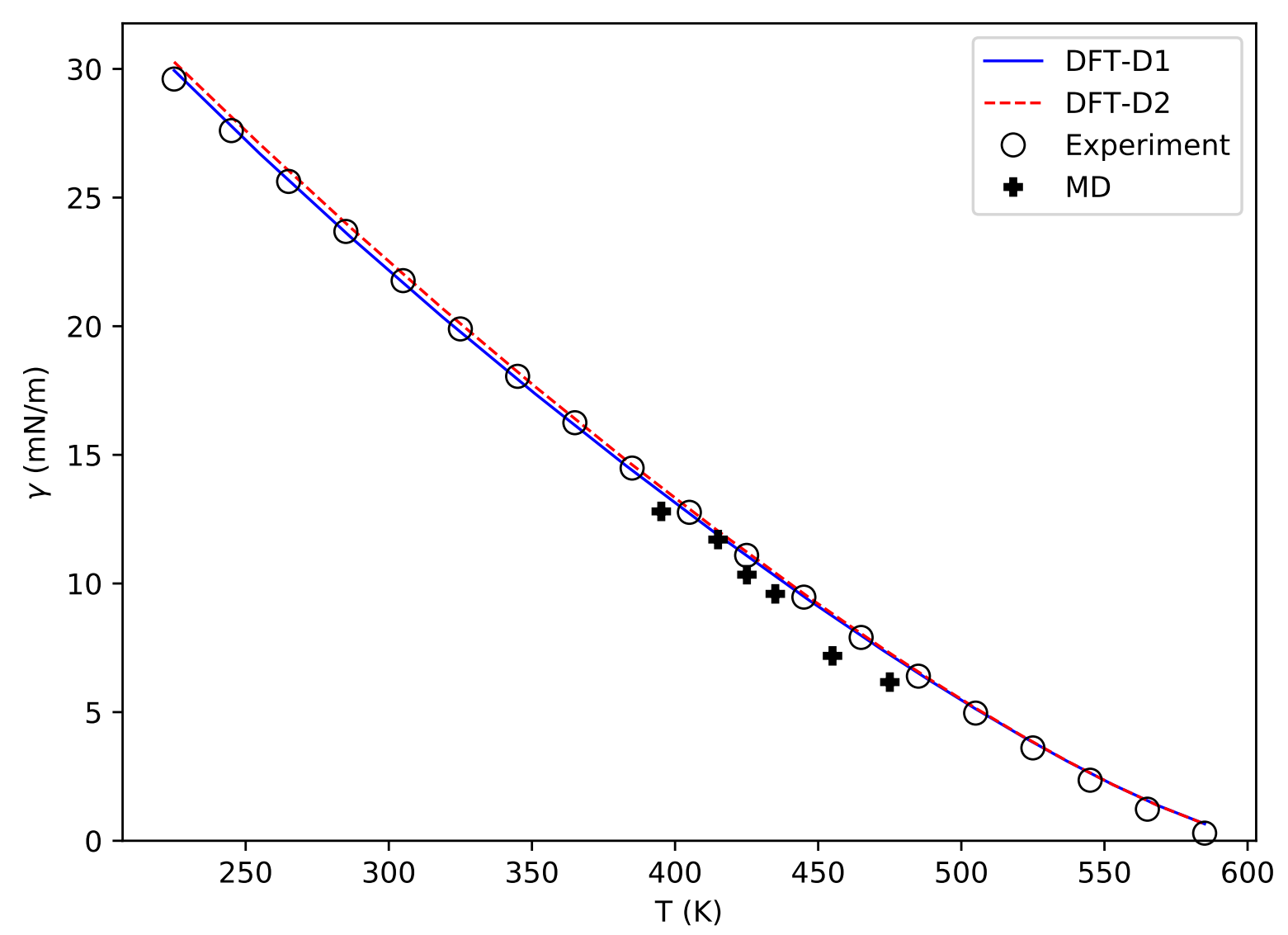

Figure 2: Interfacial tension of n-nonane calculated with DFT-D1, $\lambda=1.3862$ (solid) and DFT-D2, $\lambda=1.8738$ (dashed) compared to experimental data from Ref. 64 and results from molecular dynamics from Ref. 65.

The three models were used to calculate interfacial tension of mixtures. Firstly, we tested the performance of DFT for the mixture of n-hexane and n-decane $\left(k_{i j}=0\right)$ at 


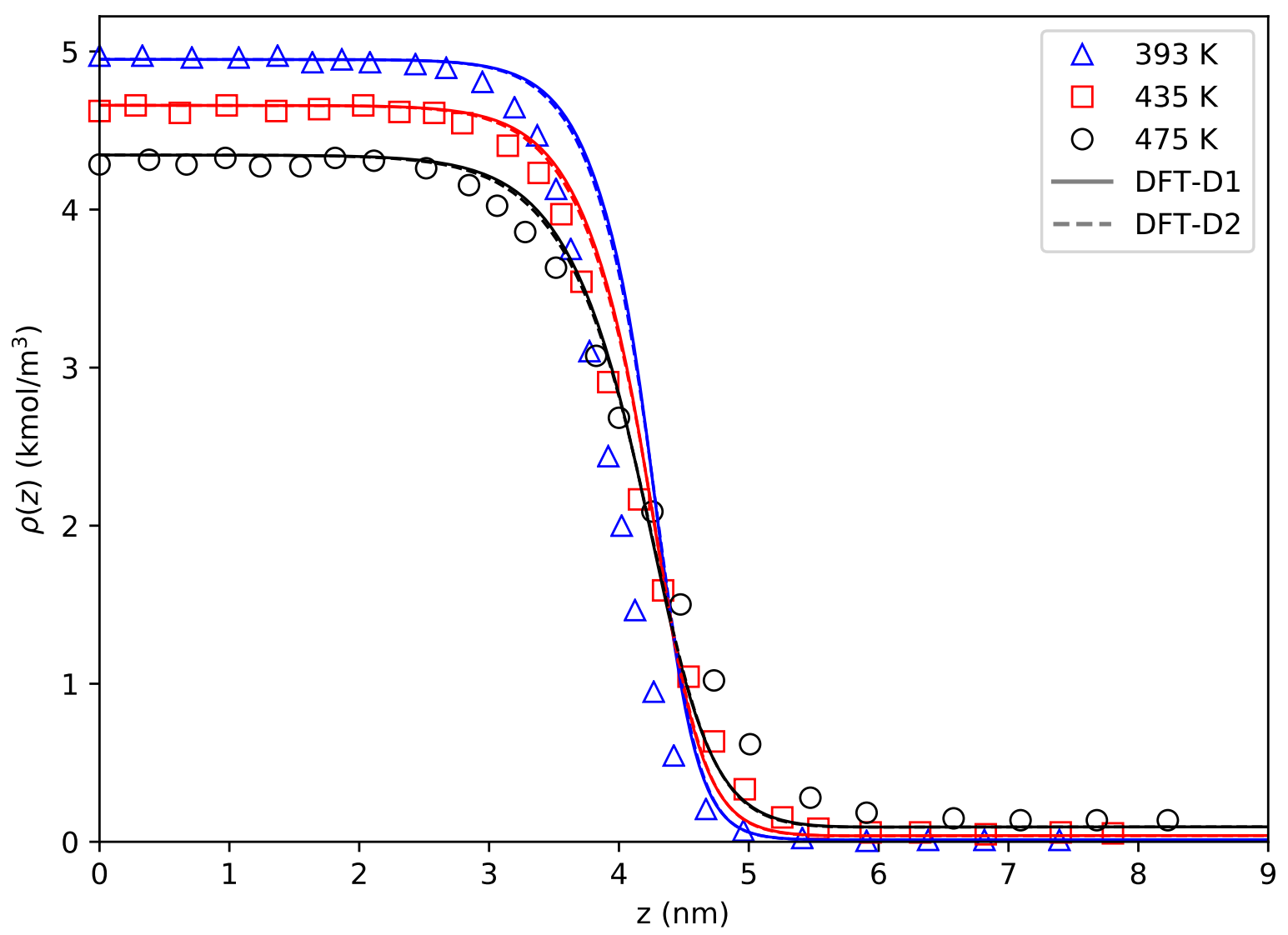

Figure 3: Density profile of the vapor-liquid interface of n-nonane calculated with DFT-D1, $\lambda=1.3862$ (solid) and DFT-D2, $\lambda=1.8738$ (dashed) compared to molecular dynamics from Ref. 65 for three temperatures. 
313.15 K, where experimental data and results from simulations of molecular dynamics were available. ${ }^{66}$ All the results are depicted in the three panels shown in Fig. 4. Panel (a) shows the results of interfacial tension, where there is good agreement between DFT, molecular dynamics and the experimental data. It can be seen that all the calculation give accurate values for the interfacial tension of the pure compounds, but DFT-D2 and DFTD3 have a superior performance for this mixture. The density profiles of both components are plotted in panels (b) and (c) and compared to molecular dynamics. All three DFT approaches give almost identical density distributions for the three compositions investigated, that differ slightly from the simulation results. It is worth noting that both DFT and molecular simulations allow the determination of the density profiles that are not available from experimental procedures, therefore, offering an insight into the structure of fluid, where DFT has the advantage of offering the same degree of accuracy as molecular simulations with only requiring a fraction of the computation time and complexity. In the following, only DFT-D1 and DFT-D2 will be used to test more systems as the performance between the 'locals' DFT-D2 and DFT-D3 is almost identical.

In Fig. 5 and Fig. 6 are shown the interfacial tension of the mixtures methane-nitrogen with a $k_{i j}=0.0307^{32}$ and methane-carbon monoxide with a $k_{i j}=0.0180^{36}$ at $83.82 \mathrm{~K}$. For these two mixtures of nearly spherical molecules, the calculated values agree very well with the experimental data. With some inspection from the figures, it can be seen that DFT-D2 performs better than DFT-D1, as we also found in the mixture of n-hexane with n-decane.

We further explore mixtures of methane with larger n-alkanes, such as propane, pentane and decane. The values of the binary interaction parameter for the mixture methane-propane is $k_{i j}=0.0$, and for methane-pentane $k_{i j}=0.024$ from Ref. 59. For the system of methanedecane $k_{i j}=0.0194$, which was fitted to VLE data from Ref. 68. From Fig. 7 we found that for these three systems, DFT-D2 just shows a slightly better performance than DFT-D1 in comparison to the other mixtures studied. Nontheless, it is shown that both models give practically identical results. 


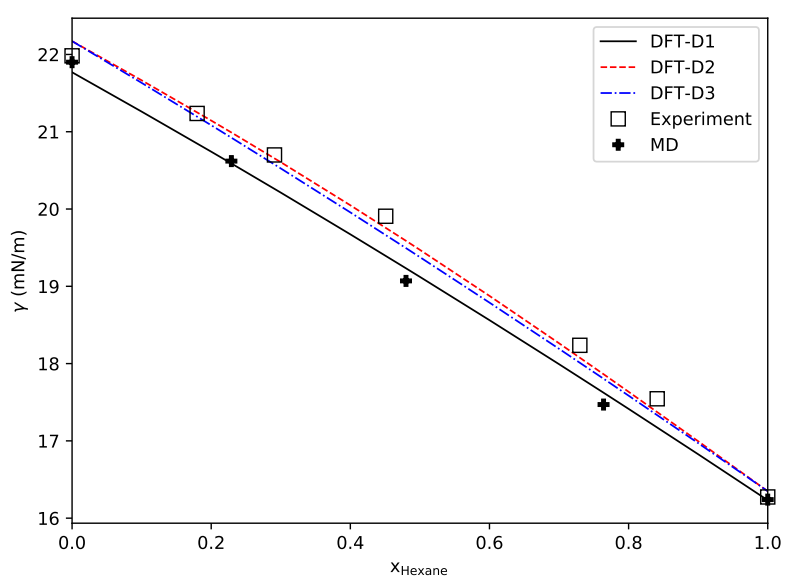

(a)

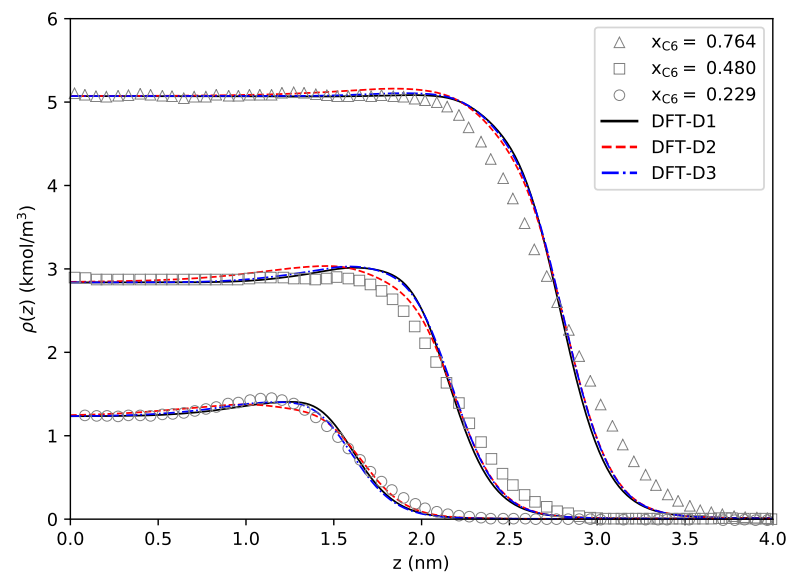

(b)

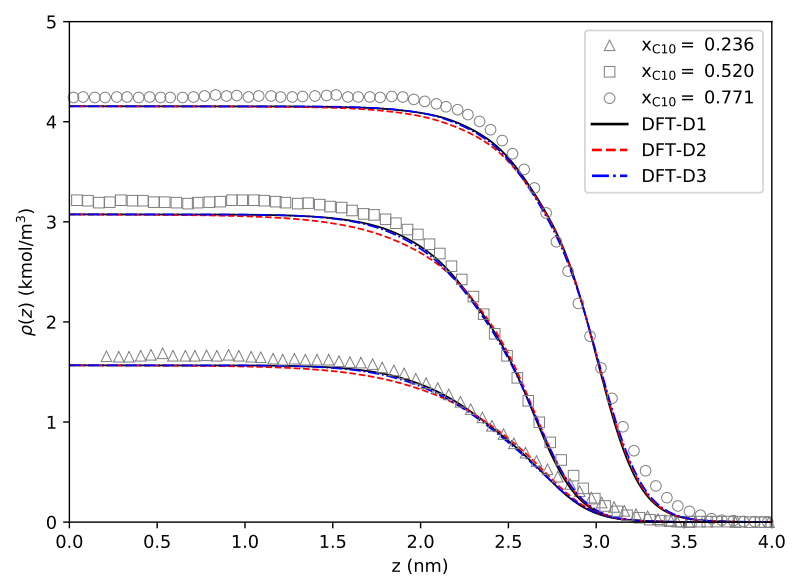

(c)

Figure 4: Interfacial tension of the mixture n-hexane with n-decane $\left(k_{i j}=0\right)$ at $313.15 \mathrm{~K}$ (a). Density profiles of the vapor-liquid interface of n-hexane (b) and n-decane (c) in the mixture at three different concentrations. Data calculated with DFT-D1 with $\lambda=1.3862$ and both DFT-D2 and DFT-D2 with $\lambda=1.8738$. Experimental data and simulation results are taken from Ref. 66. 


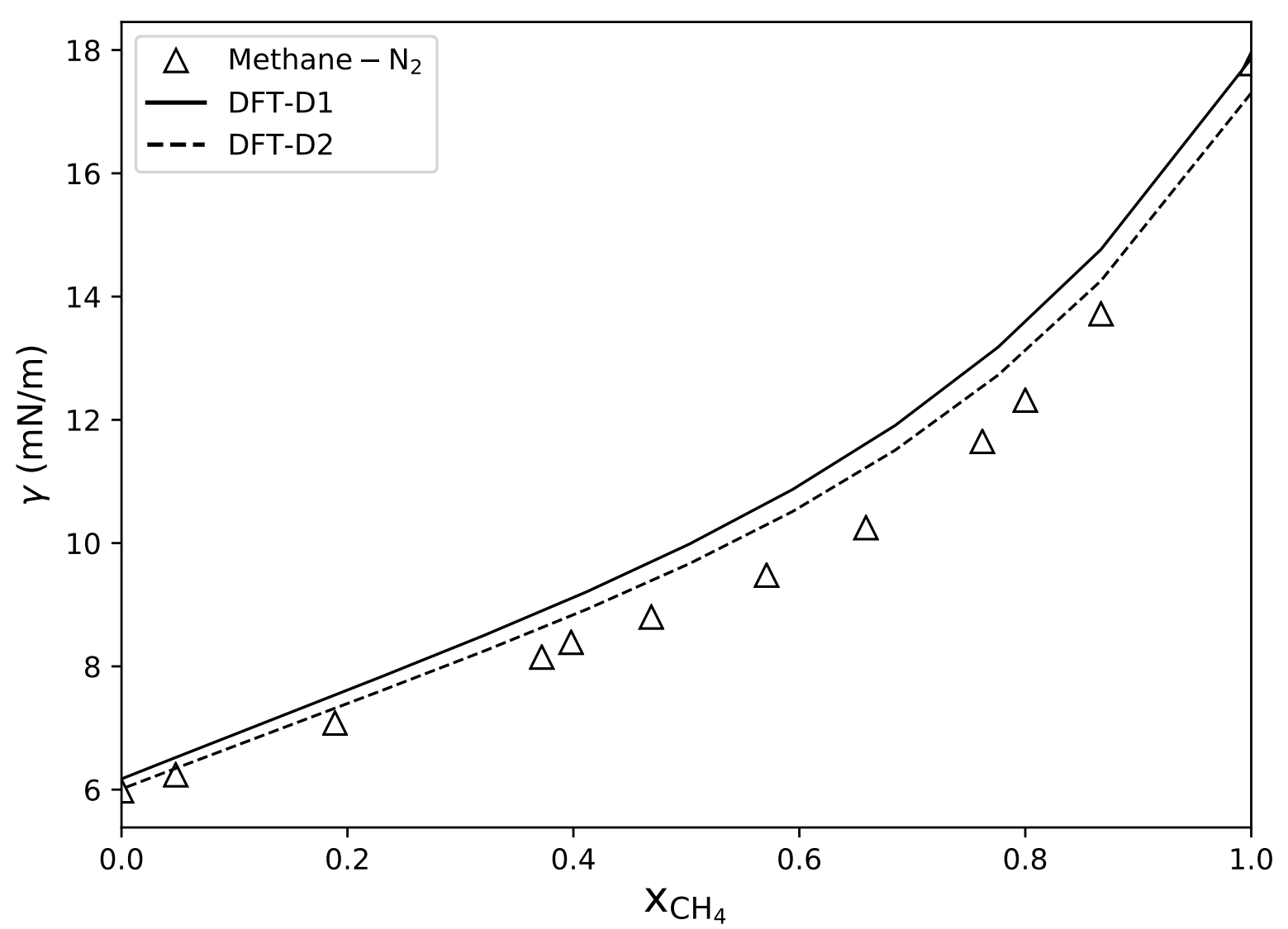

Figure 5: Interfacial tension of the vapor-liquid interface of the mixture methane-nitrogen $\left(k_{i j}=0.0307\right)$ at $83.82 \mathrm{~K}$ calculated with DFT-D1, $\lambda=1.3862$ (solid) and DFT-D2, $\lambda=1.8738$ (dashed line). Symbols are experimental data from Ref. 67. 


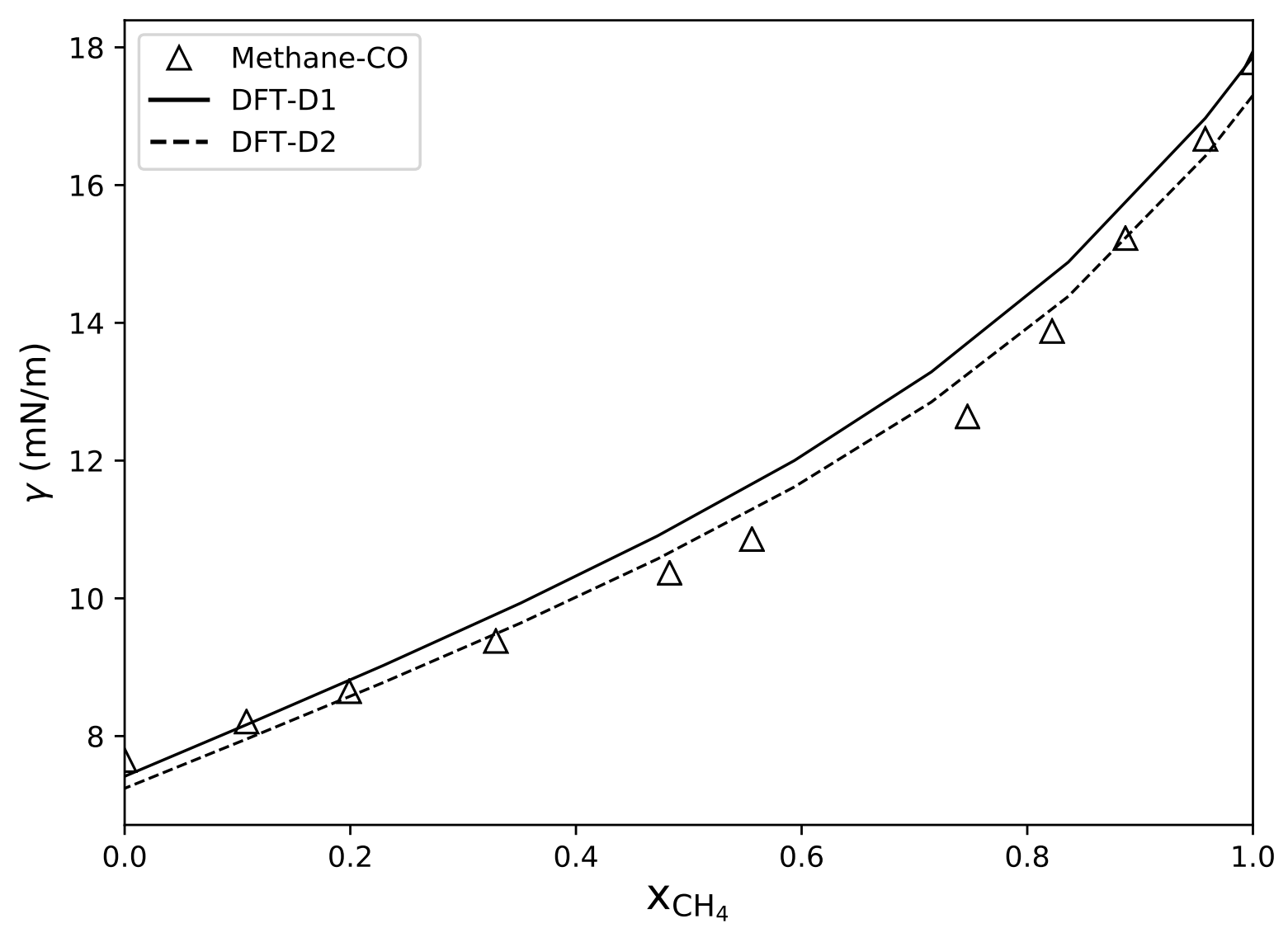

Figure 6: Interfacial tension of the vapor-liquid interface of the mixture methane-carbon monoxide $\left(k_{i j}=0.0180\right)$ at $83.82 \mathrm{~K}$ calculated with DFT-D1, $\lambda=1.3862$ (solid) and DFTD2, $\lambda=1.8738$ (dashed line). Symbols are experimental data from Ref. 67. 


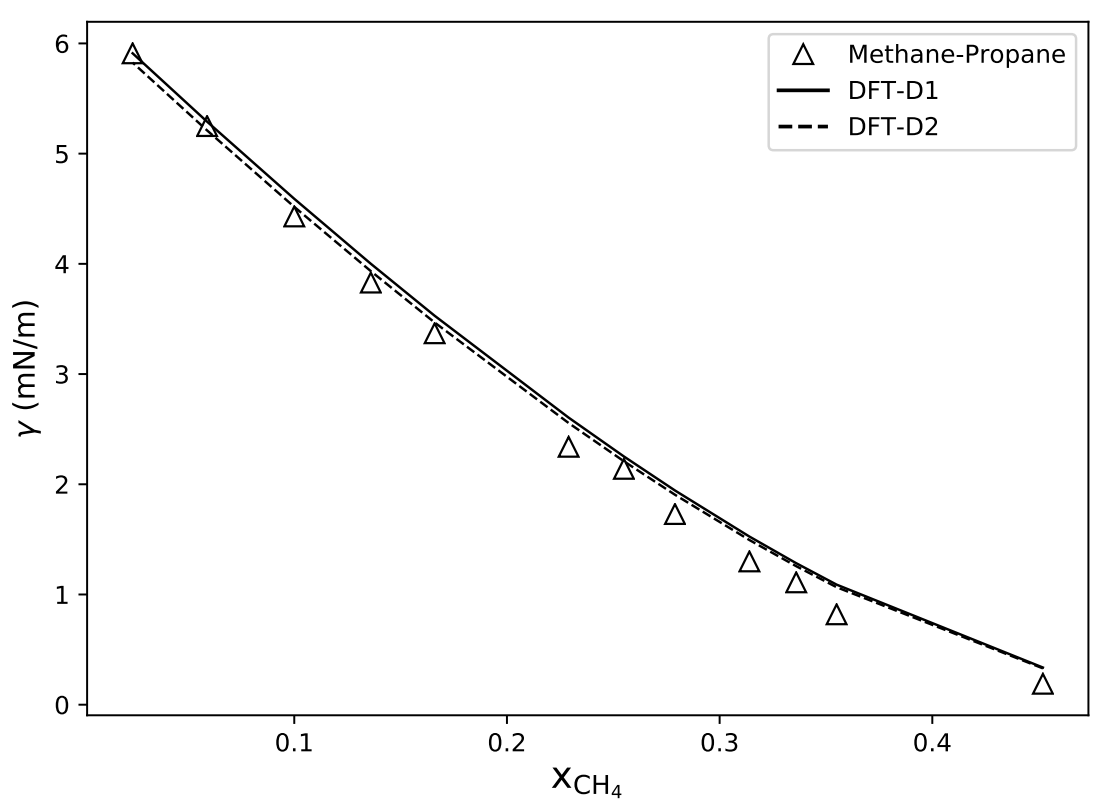

(a)

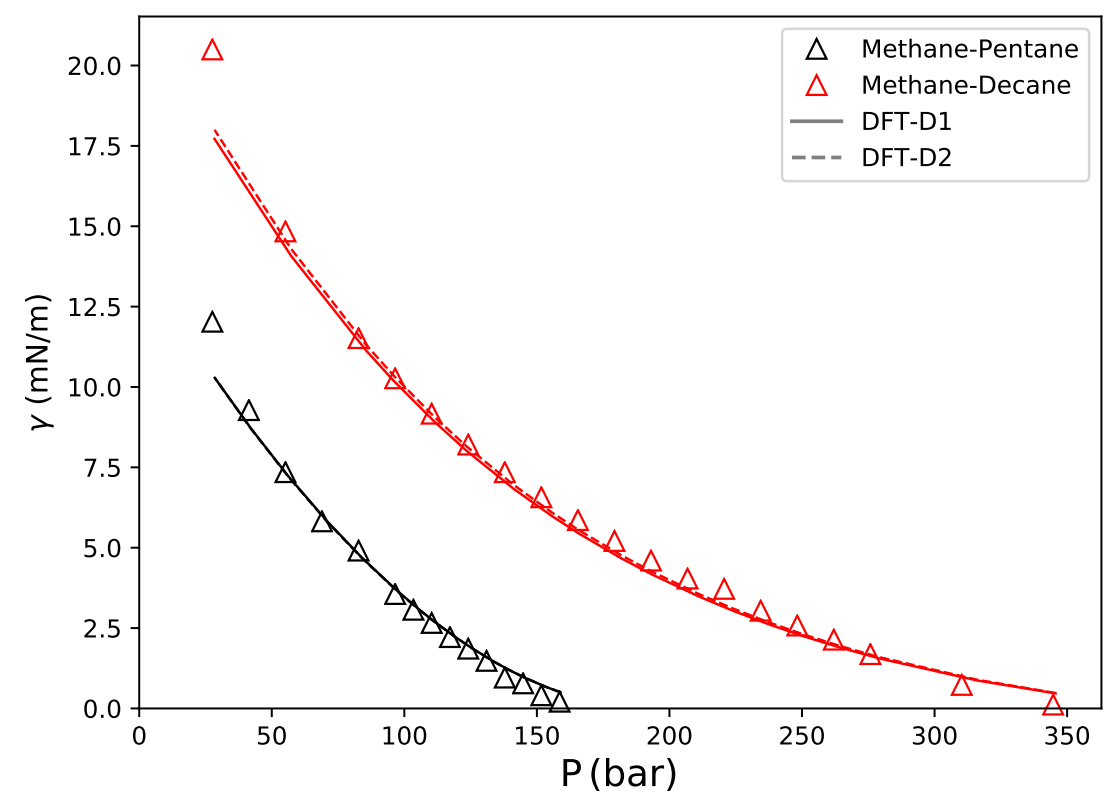

(b)

Figure 7: Interfacial tension of the vapor-liquid interface of the mixture methane-propane $\left(k_{i j}=0.0\right)$ at $303.15 \mathrm{~K}(\mathrm{a})$, and the mixtures (b) methane-pentane $\left(k_{i j}=0.024\right)$ and methane-decane $\left(k_{i j}=0.0194\right)$ at $310.93 \mathrm{~K}$. Data calculated with DFT-D1, $\lambda=1.3862$ (solid) and DFT-D2, $\lambda=1.8738$ (dashed). Symbols are experimental data taken from Ref. 69 (a), and Ref. 70 (b). 


\section{Adsorption}

In this section, the adsorption of pure gases and some of their mixtures on activated carbons is studied with both approaches DFT-D1 and DFT-D2. Our goal in this section is to study the extension of both models with their respective values of the global parameter $\lambda$ from the previous section to the calculation of adsorption. For the case of adsorption of pure gases three parameters must be fitted according to equations 27 and 28, the average pore size $H$, the specific area of the solid $A^{*}$, and the energy interaction $\varepsilon_{s f, i}$ between the molecules of the gas and the particles of the solid. For mixtures of gases, the average pore size and the specific area are considered to be the same for all components to be studied, and the energy parameters of the pure gases must be adjusted with the same values of $H$ and $A^{*}$. The latter gives a total of $n c+2$ adjustable parameters, depending on the number of gases to be studied. The fitting is carried out with the Levenberg-Marquardt algorithm ${ }^{71}$ by minimizing the following objective function

$$
\mathrm{Obj}=\sum_{i}^{n_{\exp }}\left(\frac{\Gamma_{i}^{c a l}-\Gamma_{i}^{e x p}}{\Gamma_{i}^{\exp }}\right)^{2}
$$

The performance of the results is expressed in terms of the relative average deviation $\mathrm{AD}(\%)$, calculated according to

$$
\mathrm{AD}(\%)=\frac{100}{n_{\text {exp }}} \sum_{i}^{n_{\text {exp }}}\left|\frac{\Gamma_{i}^{c a l}-\Gamma_{i}^{e x p}}{\Gamma_{i}^{e x p}}\right|
$$

Adsorption of pure methane at supercritical conditions at pressures up to $12 \mathrm{MPa}$ on activated carbons $\mathrm{K} 02^{72}$ and $\mathrm{AX}-21^{73}$ are shown in Fig. 8 and Fig. 9, respectively. The adsorption parameters for activated carbon K02 were obtained by fitting only at the middle temperature of $303 \mathrm{~K}$, and the subsequent temperatures were calculated with the fitted parameters. Similarly, the parameters for the second activated carbon AX-21 were obtained from the isotherm at $273.15 \mathrm{~K}$. The resulting parameters and their deviations are shown in Table 1. Both DFT approaches give excellent agreement with the experimental data at all 
temperatures. For AC K02 some deviations are present at the lowest temperature, whereas for AC AX-21 deviations are present at higher temperatures. Moreover, the performance of both model are practically identical for AC AX-21 including the values of the fitted parameters. For the case of AC K02, DFT-D2 shows larger deviations at the lowest temperature. It is not surprising that the results look so similar, and that is because during the fitting procedure the differences are absorbed by the resulting values of the adsorption parameters. These two systems were also studied by Monsalvo and Shapiro ${ }^{57}$ with MPTA, and by Shen et al. $^{32}$ with their hybrid DFT-PC-SAFT. For AC K02, MPTA coupled with sPC-SAFT ${ }^{74}$ gives an $\mathrm{AD}$ of $1.98 \%$, which is slightly higher than our implementation. In the other hand, the hybrid DFT-PC-SAFT is similar to DFT-D2 with a fixed value of $\lambda=1.5$, and its results show and AD of $2.10 \%$. From the latter, we can affirm that there is a small but positive improvement when fitting $\lambda$ to interfacial tension data. Moreover, for AC AX-21 MPTA gives an AD of $3.10 \%$, whereas DFT-D1 and DFT-D2 give smaller deviations of 1.20 and $1.12 \%$, respectively. The values that we obtained for the specific area of the solid do not agree with the reported values from experiments. For AC K02 the reported value is 960 $m^{2} / g^{72}$ and for AC AX-21 $3106 m^{2} / g .^{73}$

Table 1: Adsorption parameters for pure methane fitted to experimental data and deviations.

\begin{tabular}{lccccc} 
Model & $\mathrm{H}(\AA)$ & $\varepsilon_{s f} / k_{B}(\mathrm{~K})$ & $\mathrm{A}^{*}\left(m^{2} / g\right)$ & $\mathrm{AD}(\%)$ & $\operatorname{Ref}$ \\
\hline \multicolumn{5}{c}{$C H_{4} / \mathrm{AC} \mathrm{K02}$} \\
DFT-D1 & 16.25 & 86.13 & 730.36 & 1.02 & 72 \\
DFT-D2 & 16.93 & 90.81 & 689.77 & 1.23 & \\
\multicolumn{5}{c}{$C H_{4} / \mathrm{AC} \mathrm{AX}-21$} \\
DFT-D1 & 17.54 & 79.19 & 1735.0 & 1.20 & 73 \\
DFT-D2 & 17.79 & 80.90 & 1700.8 & 1.12 \\
\hline
\end{tabular}

Three additional systems for adsorption on activated carbon with experimental data for pure components and their mixtures were investigated. As mentioned before, the solid-fluid parameters of the pure components are fitted to experimental data of adsorption of the pure components, using the same pore size $H$ and specific area $A^{*}$. Once these parmeters are obtained, the calculation of adsorption for the mixtures is carried out as a full prediction 


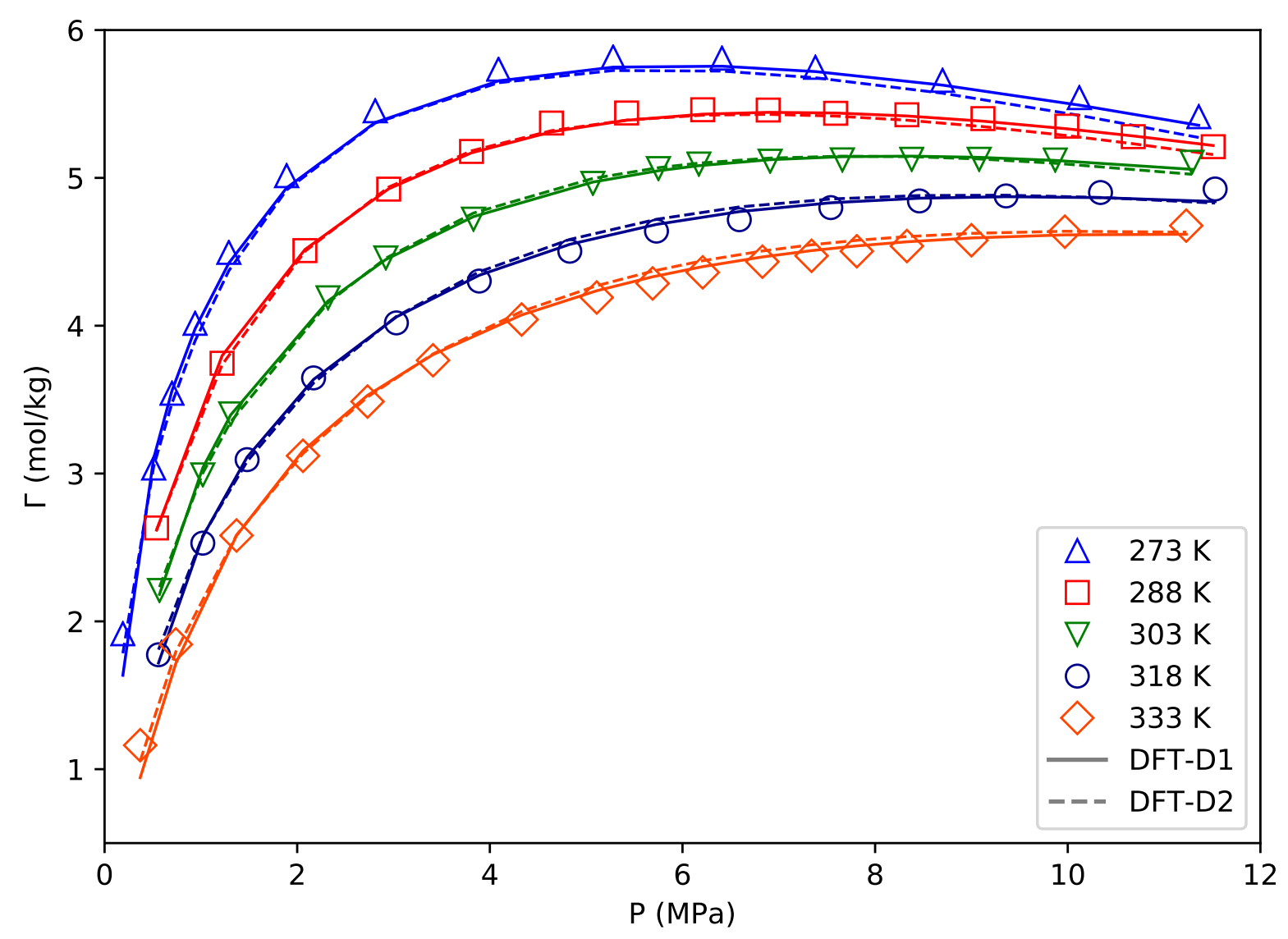

Figure 8: Adsorption of supercritical methane on AC K02 calculated with DFT-D1 and DFT-D2. The parameters were fitted only at $303.0 \mathrm{~K}$, and the other temperatures are full predictions. Symbols are experimental data from Ref. 72 . 


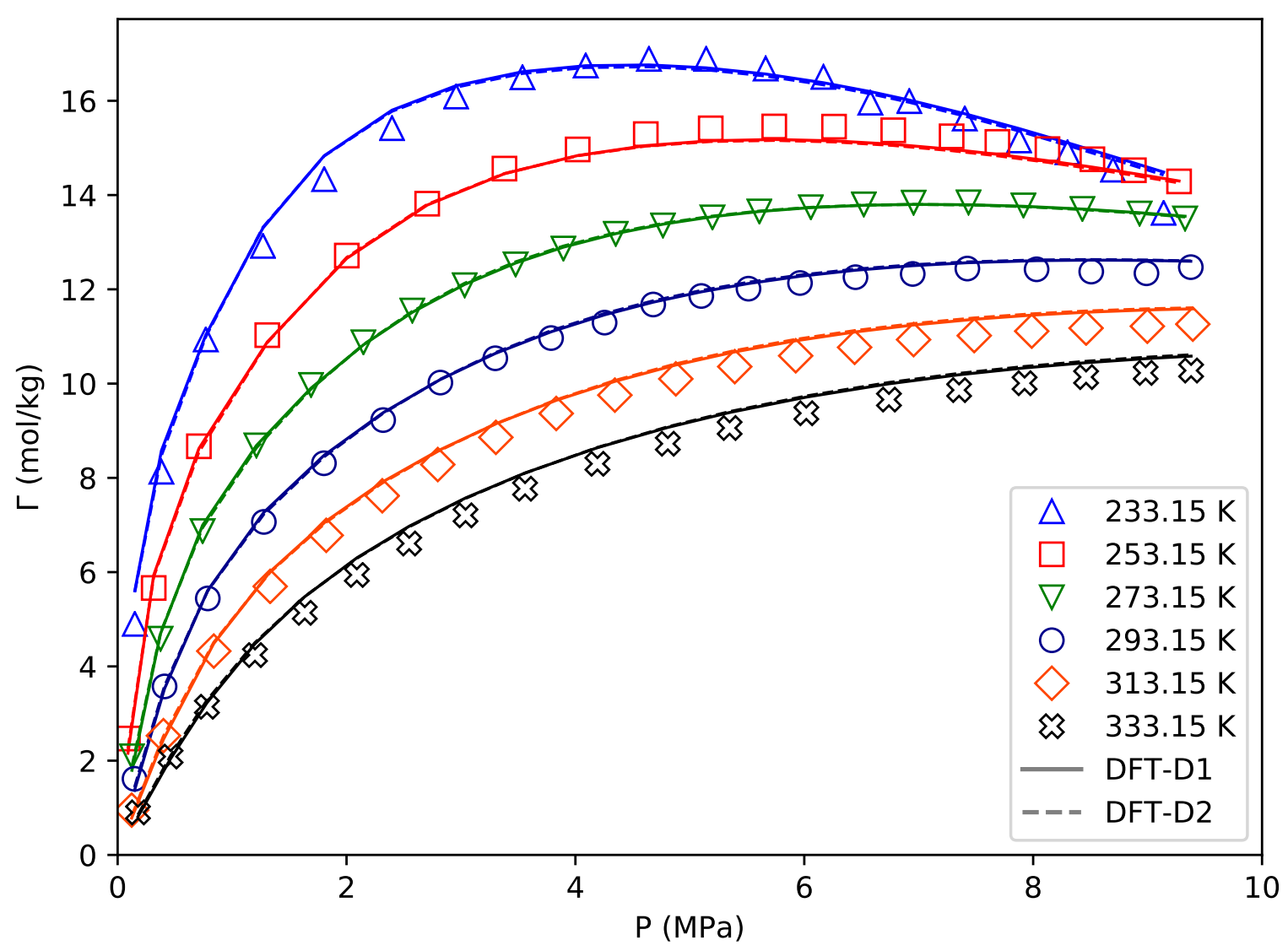

Figure 9: Adsorption of supercritical methane on AC AX-21 calculated with DFT-D1 and DFT-D2. The parameters were fitted only at $303.0 \mathrm{~K}$, and the other temperatures are full predictions. Symbols are experimental data from Ref. 73 . 
Fig. 10 shows the adsorption isotherms of methane, ethane and propane on AC Norit Row. The fitting procedure for this system was carried out with the middle temperature at 333.15 K. The lower and higher temperatures are pure predictions and show good agreement with the experimental data for both DFT approaches. The prediction of the binary mixtures 
is shown in Fig. 11. The experimental results are taken at the same temperatures of the adsorption isotherms of the pure components and plotted for different composition of the vapor (bulk phase) in equilibrium with the absorbed phase. The calculations with DFT agree with the behavior of the experimental data, where the adsorption of the heavier components is preferred over the lighter components. In addition, we find that the deviation is lower for the mixtures that contain methane, which is the opposite behavior found by Li et al. ${ }^{81}$ and their DFT coupled with the Peng-Robinson equation of state. ${ }^{82}$ Their results show larger deviation for adsorption of the methane containing mixtures.

For the second system, our implementation is tested for the adsorption of methane, nitrogen and carbon dioxide in AC Norit R1 Extra at pressures up to $6 \mathrm{MPa}$ at a temperature of 298 K. The results of the pure components are plotted in Fig. 12. Similar to the previous system the adsorption increases with the increasing pressure. The experimental data of adsorption of carbon dioxide shows a maximum at about $4 \mathrm{MPa}$. It is hard to examine from the figure as the isotherm is very flat, but our implementation predicts a maximum adsorption of $\mathrm{CO}_{2}$ at about $3 \mathrm{MPa}$. The isotherms of nitrogen and methane agree very well with experimental data, whereas carbon dioxide shows slight deviations. The latter can be attributed to the high polarity of the $\mathrm{CO}_{2}$ molecule, and additional contributions to the Helmholtz free energy functional may be taken into account to consider this type of interactions. At these conditions, carbon dioxide is quite close to its critical temperature of $304 \mathrm{~K}^{64}$ and this fact is reflected in the shape of the isotherm. There is an inflection point at about $0.5 \mathrm{MPa}$, which is characteristic of a first order vapor-liquid transition. This is a phenomenon that DFT has shown to be able to predict with the formation of a liquid-like film close to the walls that strongly depends on the pore size. ${ }^{2,83}$ However, this behavior is not shown by the experimental data of pure $\mathrm{CO}_{2}$, and the prediction of adsorption for the mixtures also show this phenomenon. The prediction results for the mixtures of these systems are plotted in Figures 13, 14 and 15. For each binary mixture, the single adsorption of each of the components is obtained by specifying its mean mole fraction in the gas in equilibrium 


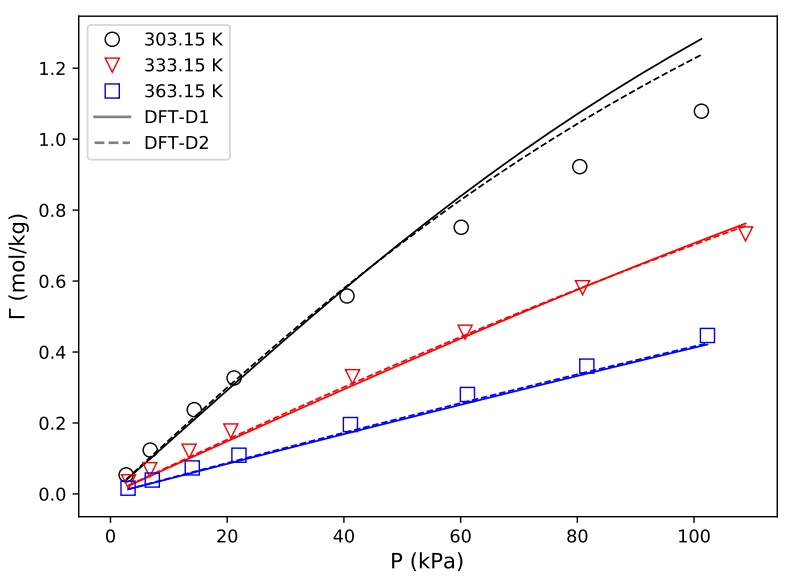

(a)

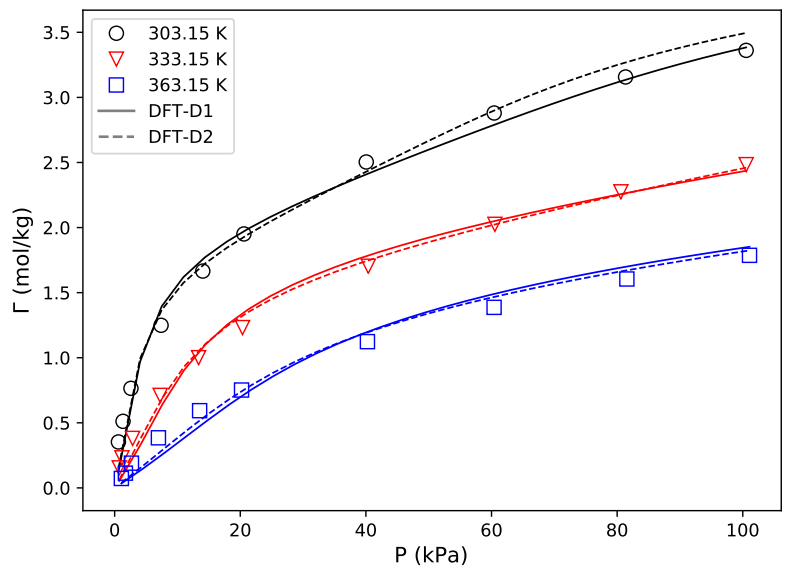

(b)

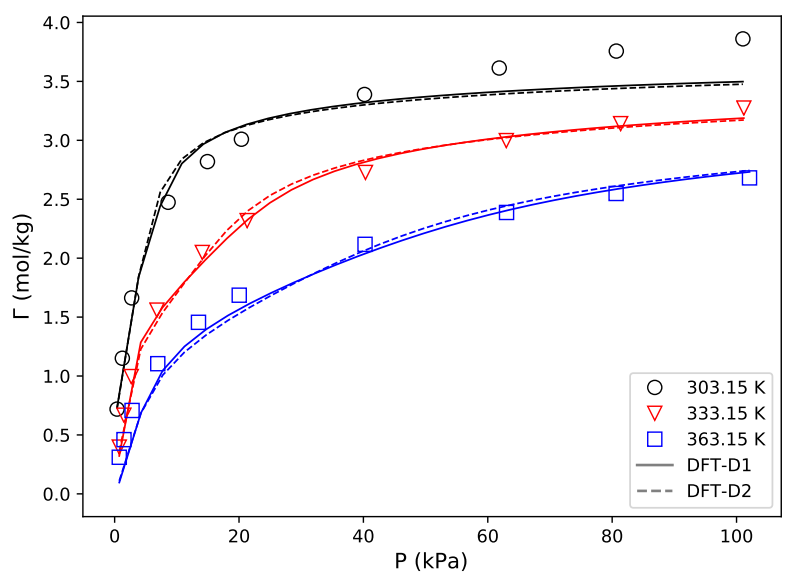

(c)

Figure 10: Adsorption of pure methane (a), ethane (b) and propane (c) at three temperatures on activated carbon AC Norit Row calculated with DFT-D1 and DFT-D2. The parameters are fitted only at $333.15 \mathrm{~K}$. Results at 303.15 and $363.15 \mathrm{~K}$ are full predictions. Symbols are experimental data from Ref. 75 . 


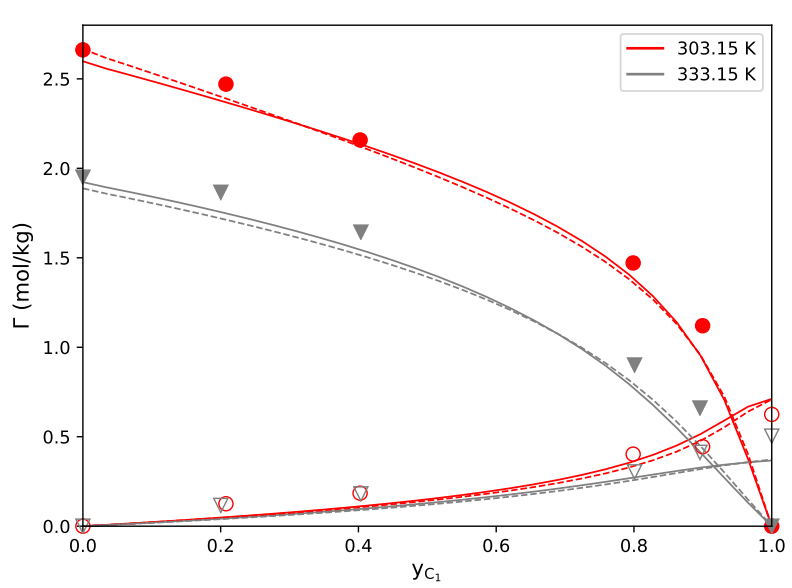

(a)

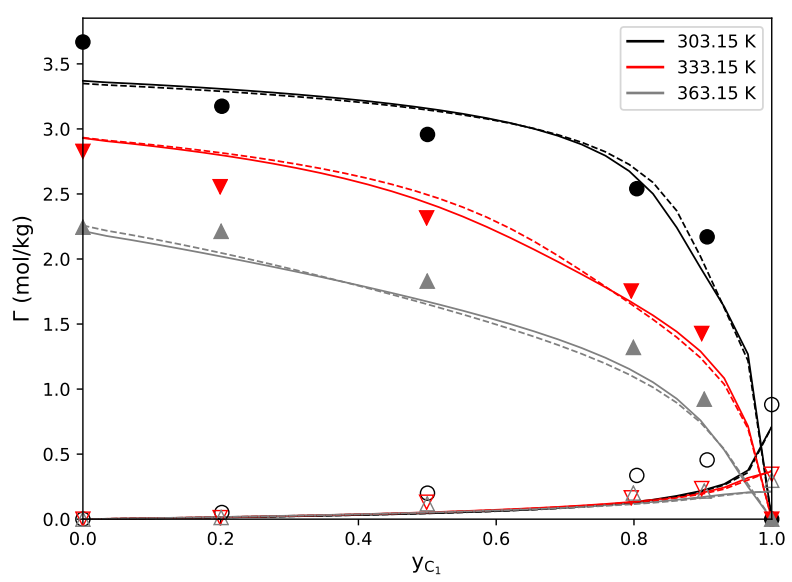

(b)

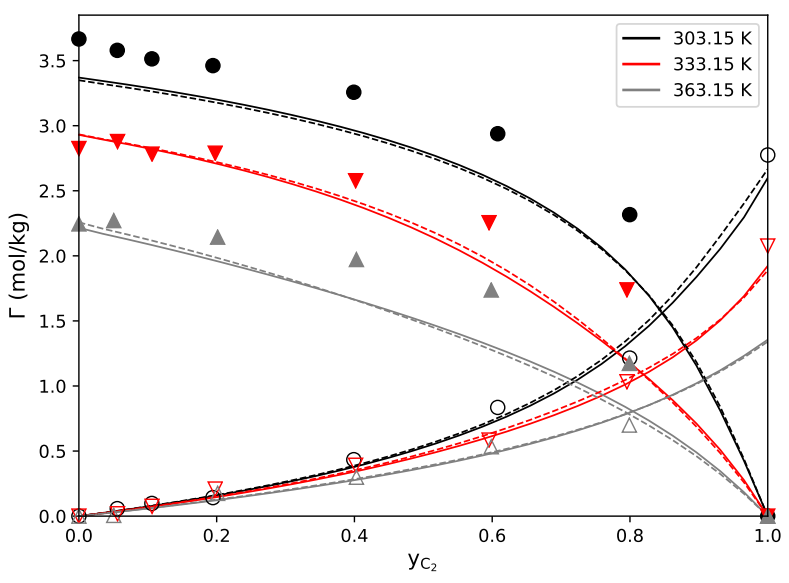

(c)

Figure 11: Prediction of adsorption of the mixtures methane-ethane $\left(k_{i j}=-0.006\right)$ (a), methane-propane $\left(k_{i j}=0.010\right)(\mathrm{b})$ and ethane-propane $\left(k_{i j}=0.0089\right)(\mathrm{c})$ at different temperatures on activated carbon AC Norit Row calculated with DFT-D1 (solid lines) and DFT-D2 (dashed lines). Solid symbols are experimental data of the heavier component. Experimental data from Ref. 75 . 
with the adsorbed amount. DFT shows good agreement with the experimental data for all binary mixtures, specially for the mixture $\mathrm{CH}_{4} / \mathrm{N}_{2}$ where the agreement is excellent. For the mixtures containing carbon dioxide, we observed a maximum predicted by DFT at the two lower concentrations for methane in panel (a) of Fig. 14, whereas for nitrogen a maximum is found in all three concentrations on panel (a) of Fig. 15. For these two mixtures carbon dioxide is the heaviest component and it shows similar behavior for both cases, reaching a limiting value of adsorption at all the concentrations studied.

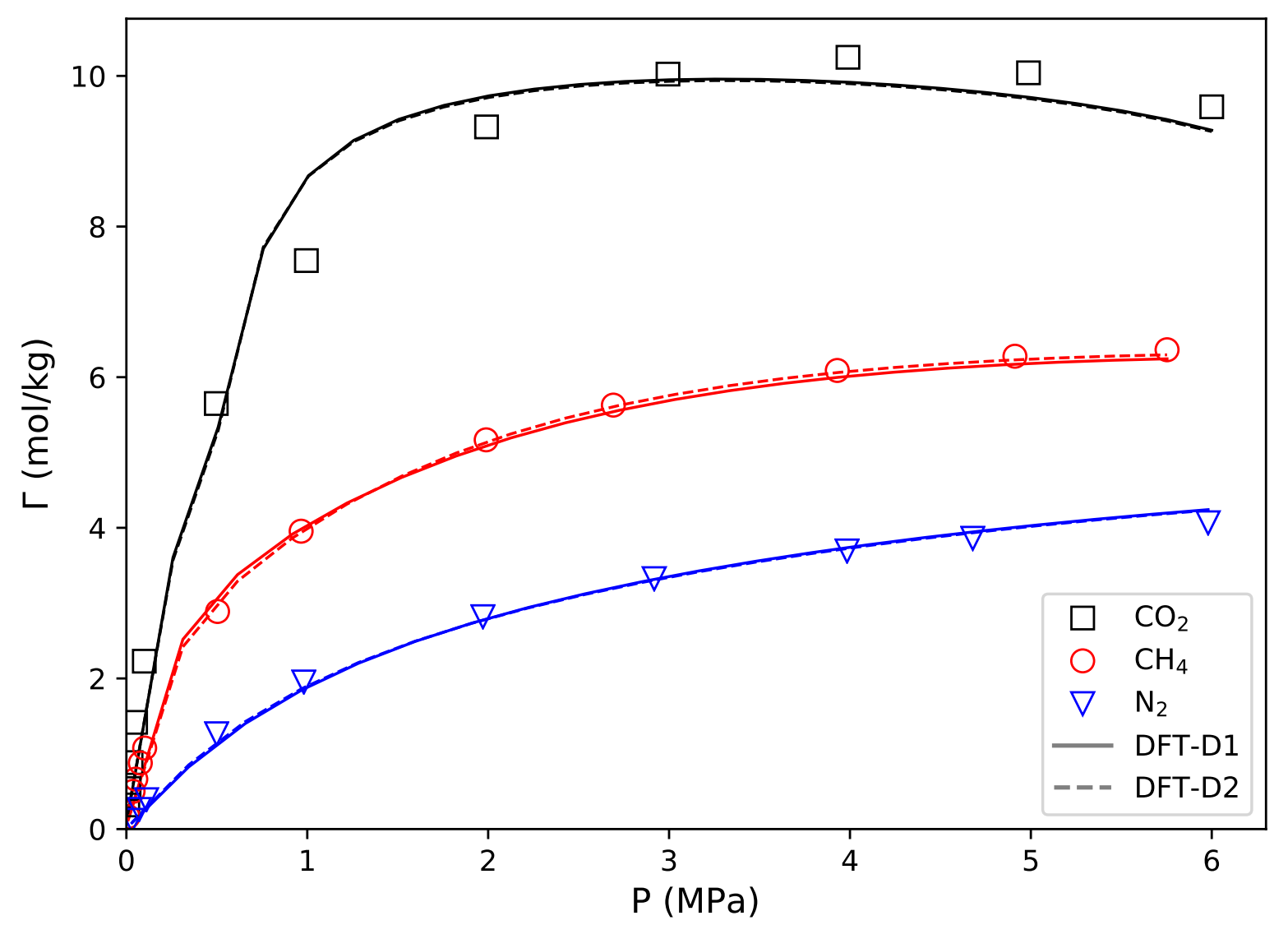

Figure 12: Adsorption of methane, nitrogen and carbon dioxide at $298 \mathrm{~K}$ on AC Norit R1 Extra calculated with DFT-D1 and DFT-D2. Symbols are experimental data from Ref. 76.

The last activated carbon tested corresponds to a system of methane, nitrogen and carbon dioxide from experimental data at pressures up to $14 \mathrm{MPa}$ and $318.2 \mathrm{~K}$ on activated carbon Calgon F400. ${ }^{77}$ This set of experimental data has been used by Monsalvo and Shapiro 


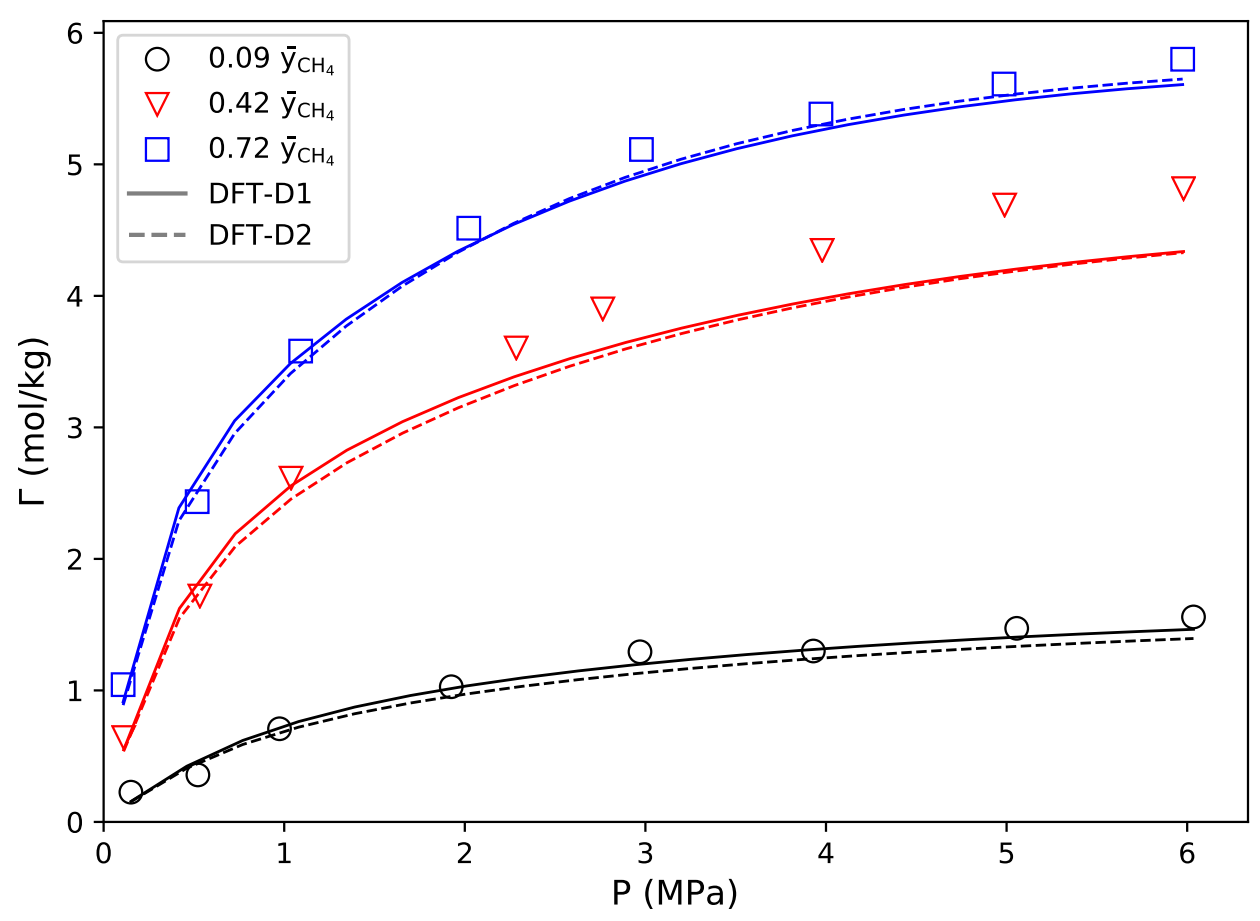

(a)

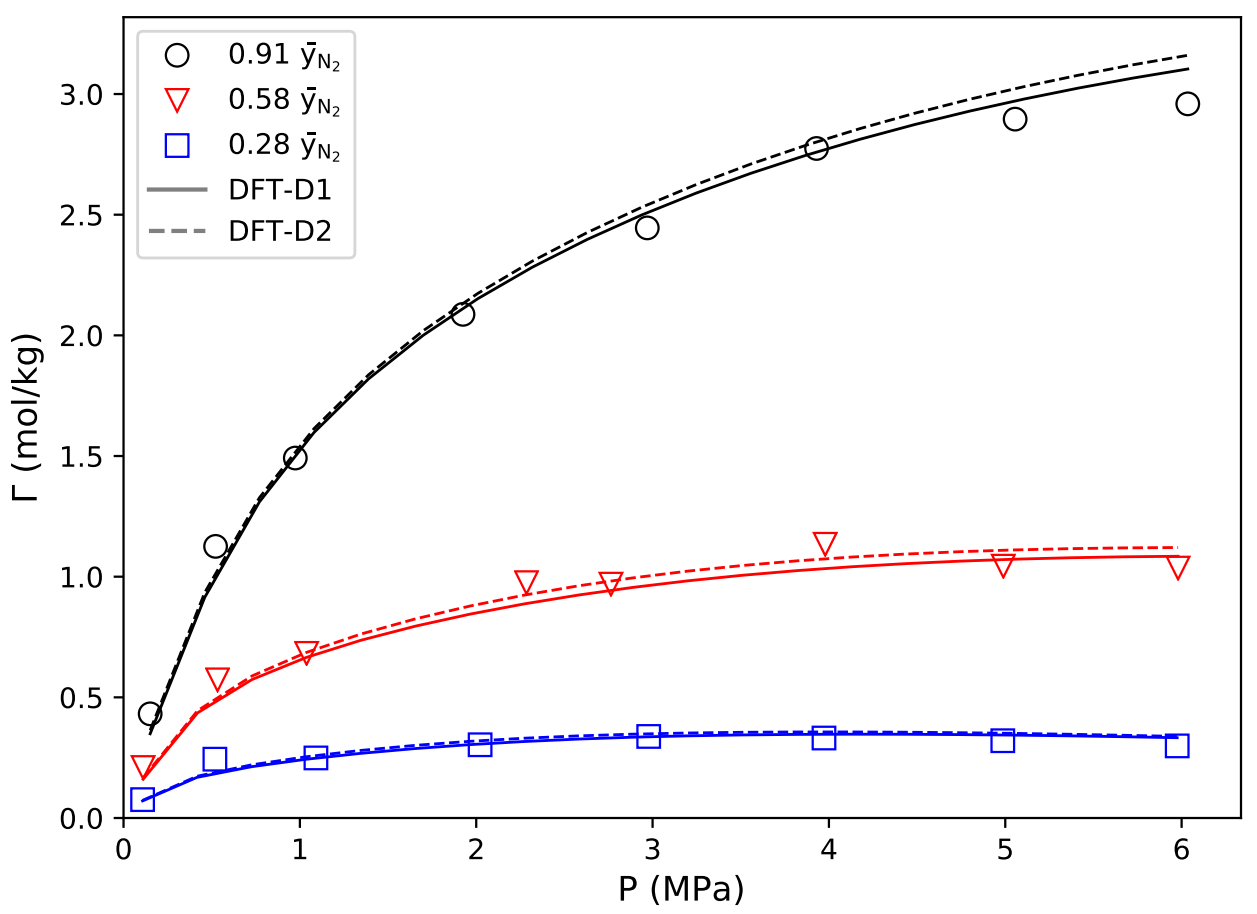

(b)

Figure 13: Prediction of adsorption of methane (a) and nitrogen (b) in the mixture methanenitrogen $\left(k_{i j}=0.0307\right)$ at $298 \mathrm{~K}$ and different average composition of the gas at equilibrium on AC Norit R1 Extra calculated with DFT-D1 and DFT-D2. Symbols are experimental data from Ref. 76 . 


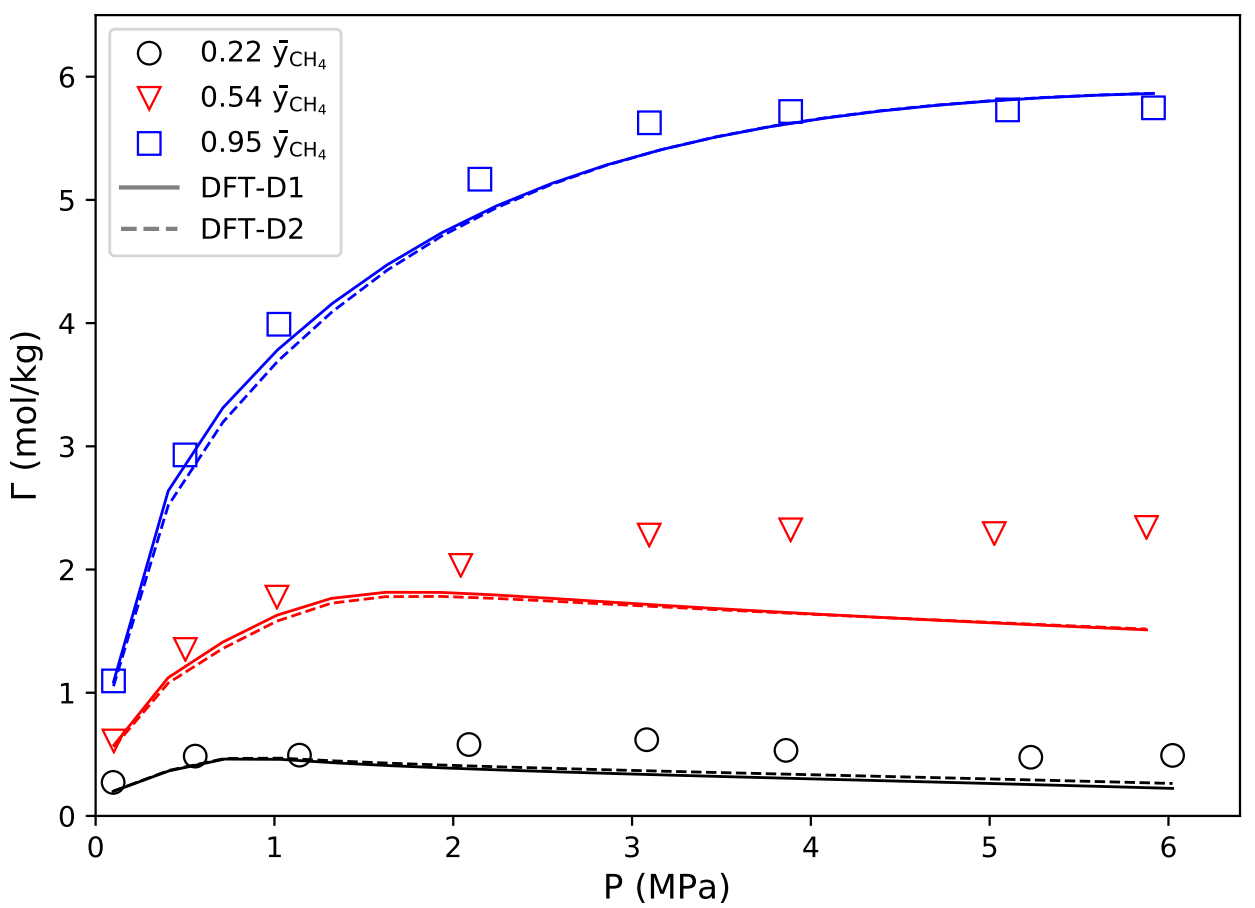

(a)

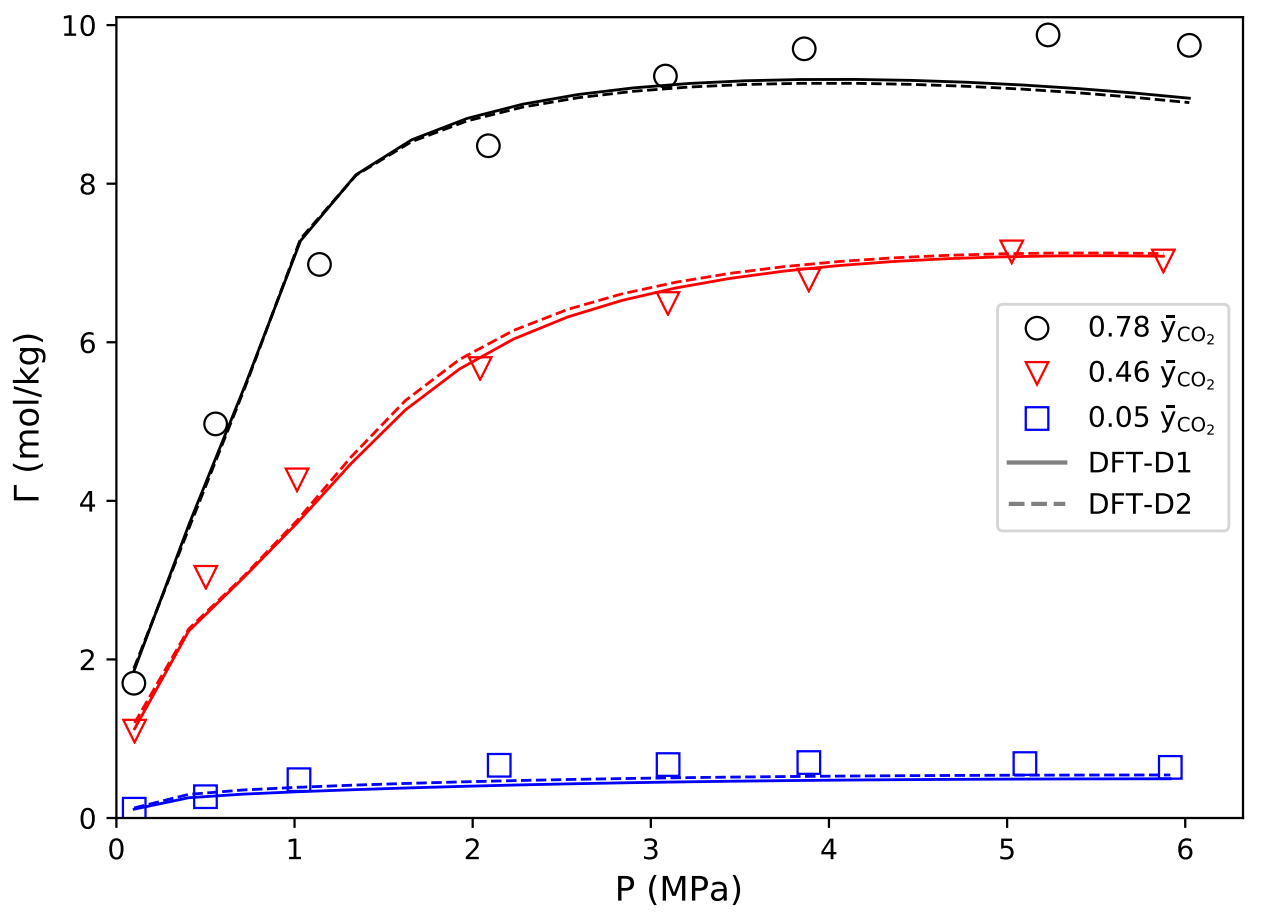

(b)

Figure 14: Prediction of adsorption of methane (a) and carbon dioxide (b) in the mixture methane-carbon dioxide $\left(k_{i j}=0.065\right)$ at $298 \mathrm{~K}$ and different average composition of the gas at equilibrium on AC Norit R1 Extra calculated with DFT-D1 and DFT-D2. Symbols are experimental data from Ref. 76 . 


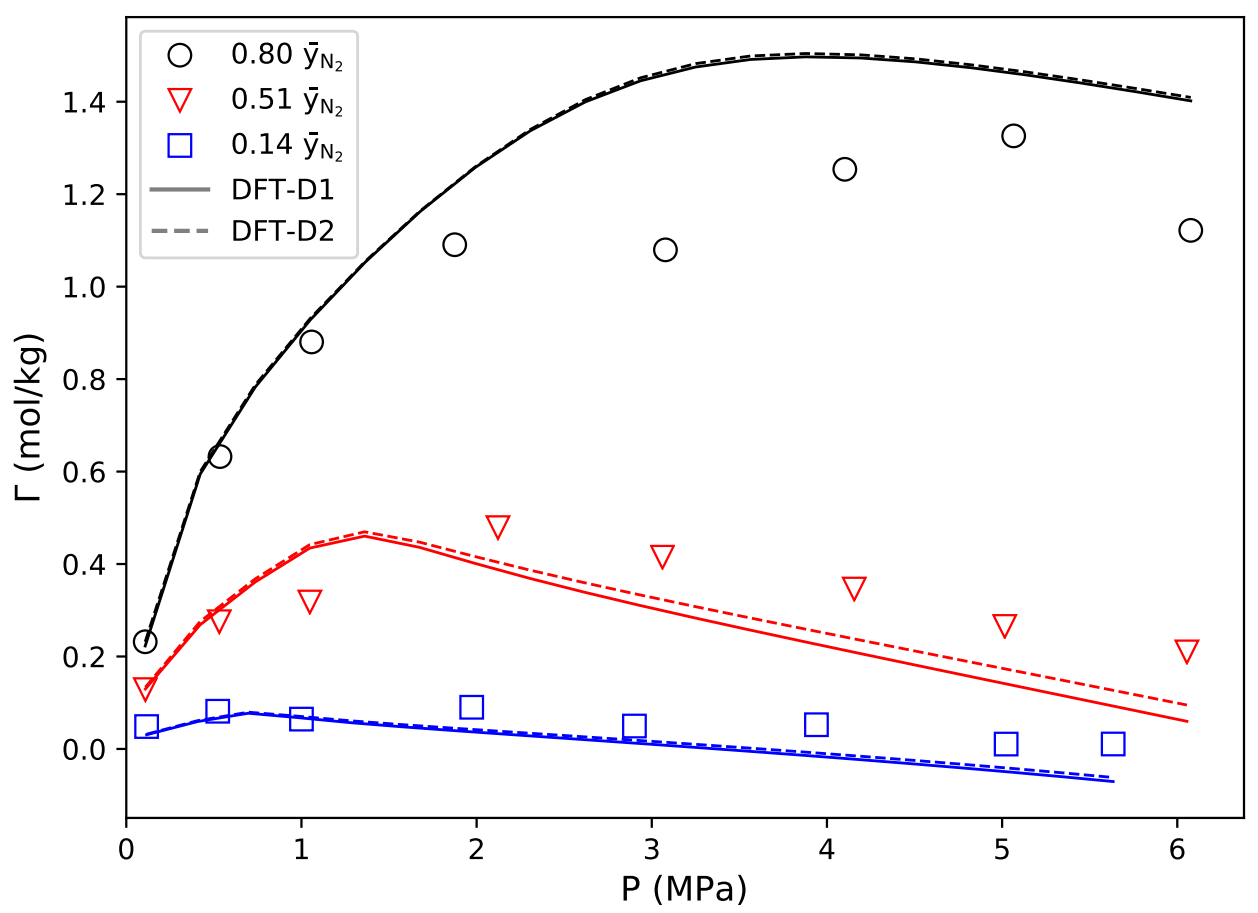

(a)

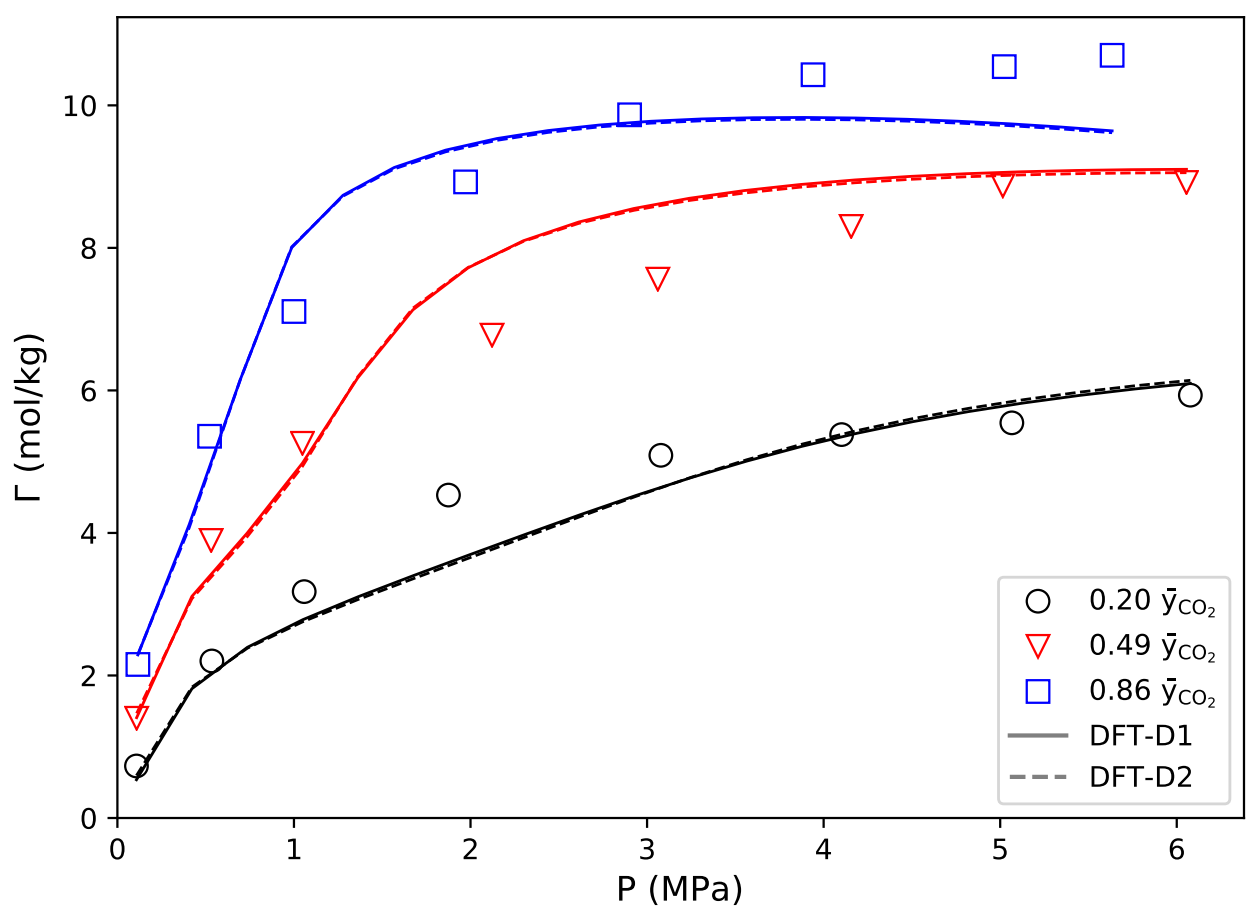

(b)

Figure 15: Prediction of adsorption of nitrogen (a) and carbon dioxide (b) in the mixture nitrogen-carbon dioxide at $298 \mathrm{~K}$ and different average composition of the gas at equilibrium on AC Norit R1 Extra calculated with DFT-D1 and DFT-D2. Symbols are experimental data from Ref. 76. 


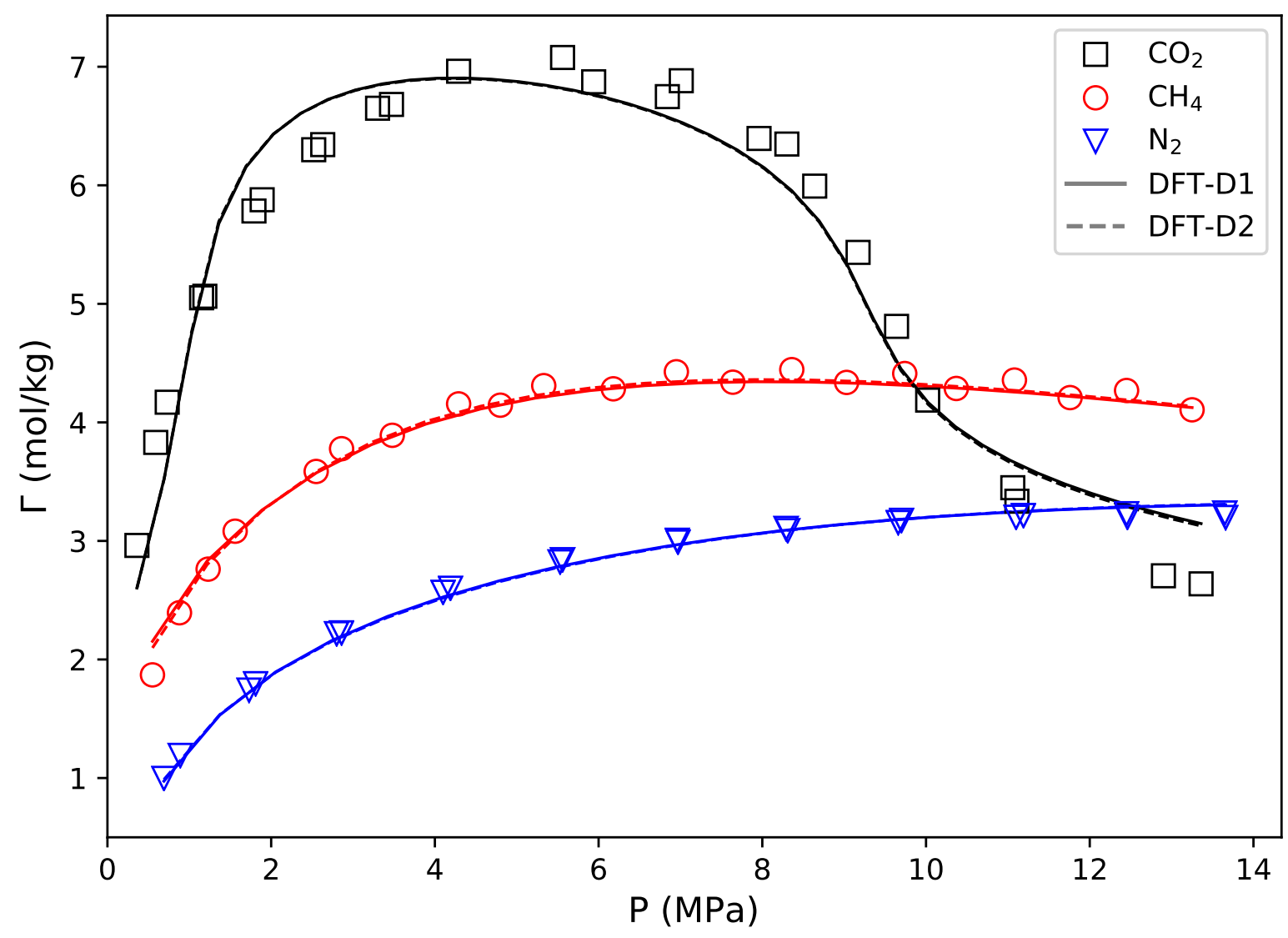

Figure 16: Adsorption of methane, nitrogen and carbon dioxide $\left(k_{i j}=-0.0055\right)$ at $318.2 \mathrm{~K}$ on AC Calgon F400 calculated with DFT-D1 and DFT-D2. Symbols are experimental data from Ref. 77. 
with MPTA, ${ }^{57}$ and by Shen et al. with their hybrid DFT-PC-SAFT. ${ }^{32}$ The resulting fitted parameters for the solid and the solid-fluid adsorption energies of the pure compounds are shown in Table 2, and the adsorption isotherms of the pure compounds are plotted in Fig. 16. These results show that the adsorption isotherms of the pure components are better correlated by DFT, both from Shen et al. and from this work, with AD $3.41 \%$ and $3.51 \%$ (DFT-D2), respectively, whereas MPTA gives a value of $4.83 \%$. Both DFT-D1 and DFT-D2 give almost identical results, similar to the previous systems studied. We further explore the prediction of adsorption of the binary mixtures of the three components with our DFT implementation. The results of adsorption for the individual components in the three mixtures $\mathrm{CH}_{4} / \mathrm{N}_{2}, \mathrm{CH}_{4} / \mathrm{CO}_{2}$ and $\mathrm{N}_{2} / \mathrm{CO}_{2}$ are shown in Figures 17-19. In the mixture $\mathrm{CH}_{4} / \mathrm{N}_{2}$, the prediction of single adsorption of methane is in excellent agreement with experimental data, whereas a larger deviation is found for nitrogen. In addition, for this binary mixture the single adsorption of nitrogen shows a maxima in the three lower concentrations that DFT is able to predict. For the other two mixtures, the agreement is fairly good and the adsorption behavior is completely captured by DFT, including the maxima shown in the corresponding systems. Moreover, the prediction of the negative adsorption for nitrogen in the system $\mathrm{N}_{2} / \mathrm{CO}_{2}$ for the lowest $\mathrm{N}_{2}$ concentration and highest pressures is well capture by our implementation. The latter means that less nitrogen is present in the adsorbed amount than it would be if it were replaced by the nitrogen present in the equilibrium bulk phase. ${ }^{77}$ In addition, it should be noticed that Shen et al. ${ }^{32}$ and Monsalvo and Shapiro ${ }^{57}$ calculated the adsorption different from our work. They defined different limits of integration for equation Eq. 28, that change the volume accessible to the particles. Shen et al. used a value of $0.5 \sigma_{\mathrm{s}}$ and Monsalvo and Shapiro used $0.7 \sigma_{\mathrm{sf}, \mathrm{i}}$. The reason for these selections were not deeply discussed by the authors, and as they introduce an additional level of empiricism we opted to use the limits as shown in equation Eq. 28 to take into account the volume excluded by the particles of the solid and the fluid. 


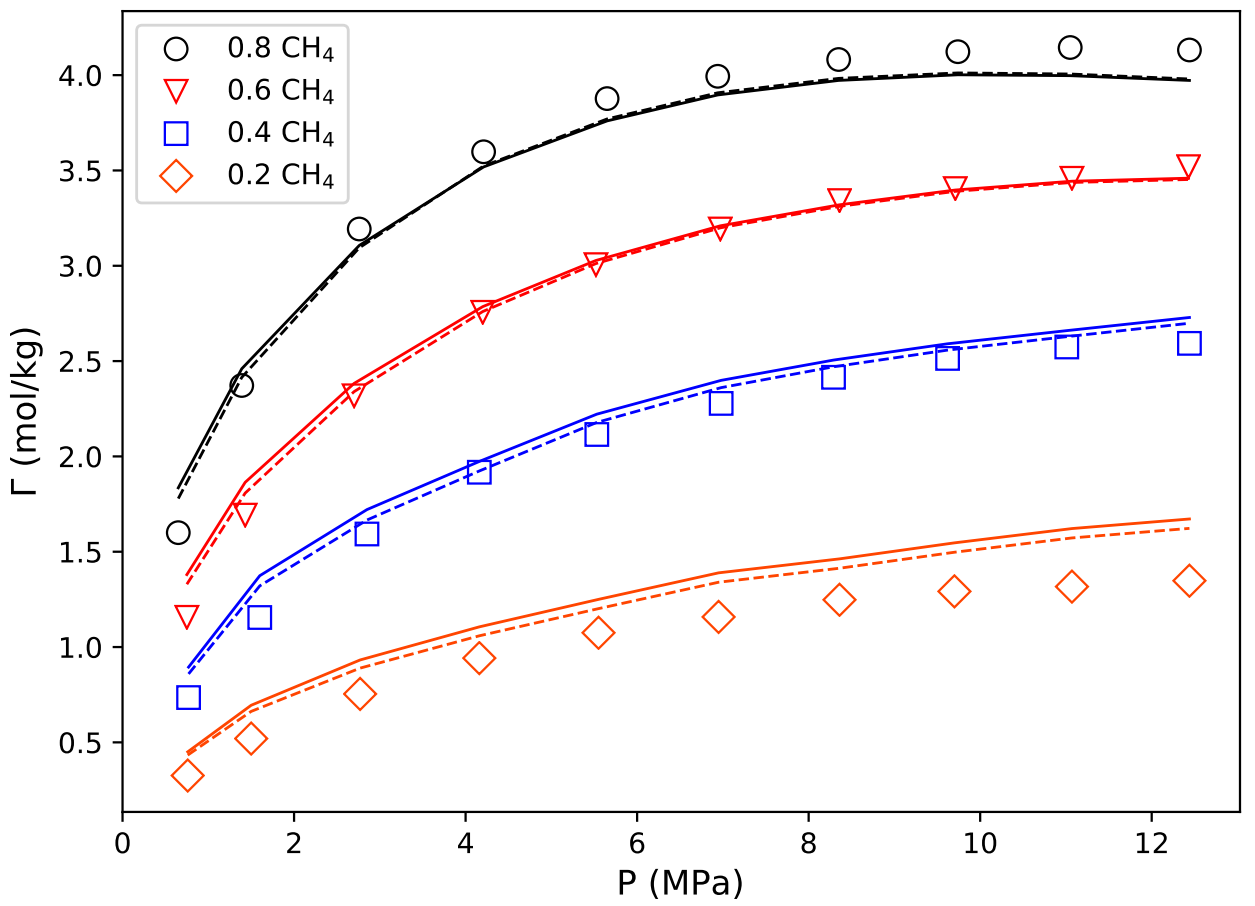

(a)

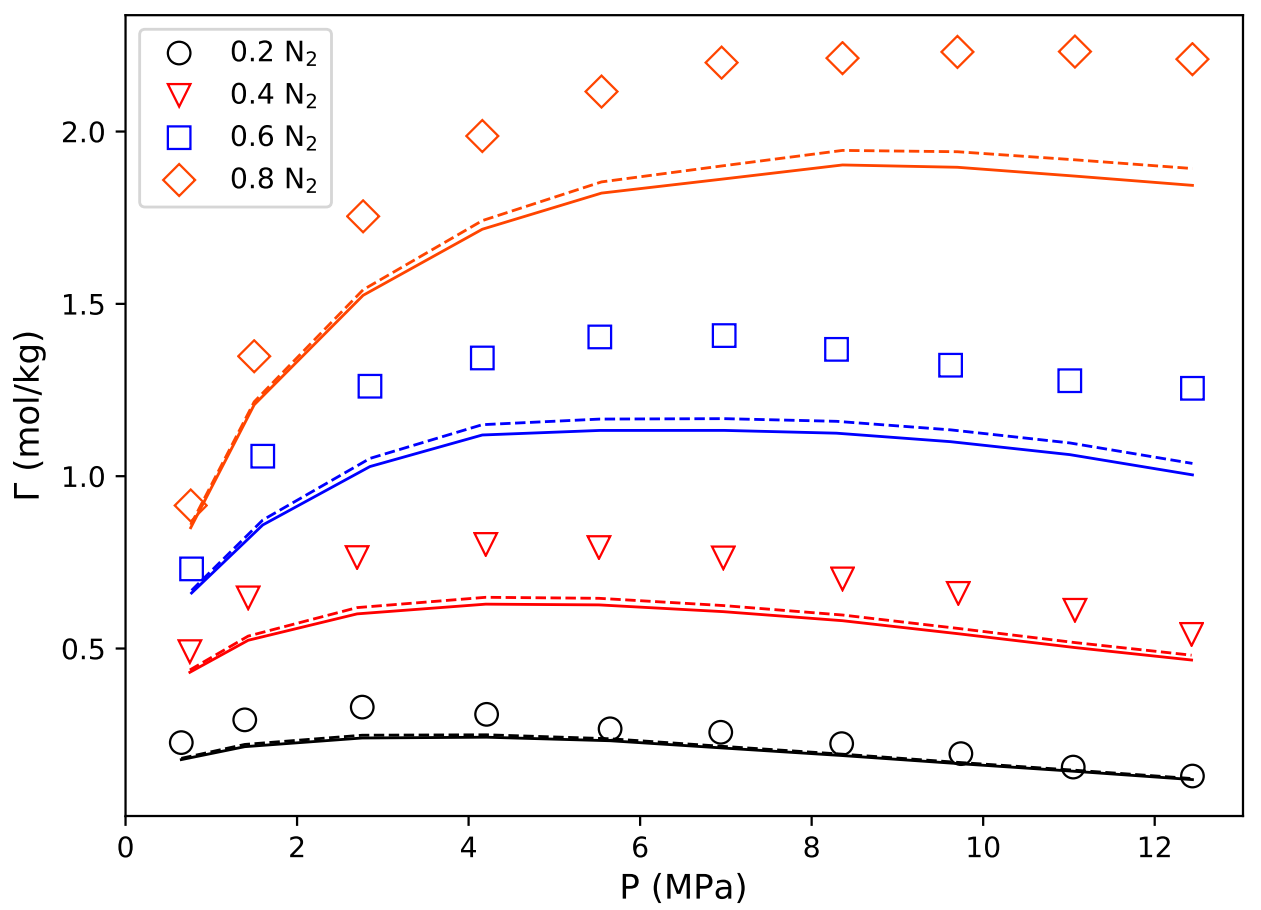

(b)

Figure 17: Prediction of adsorption of methane (a) and nitrogen (b) in the mixture methanenitrogen $\left(k_{i j}=0.0307\right)$ at $318.2 \mathrm{~K}$ on AC Calgon F400 calculated with DFT-D1 and DFT-D2. Symbols are experimental data from Ref. 77. 


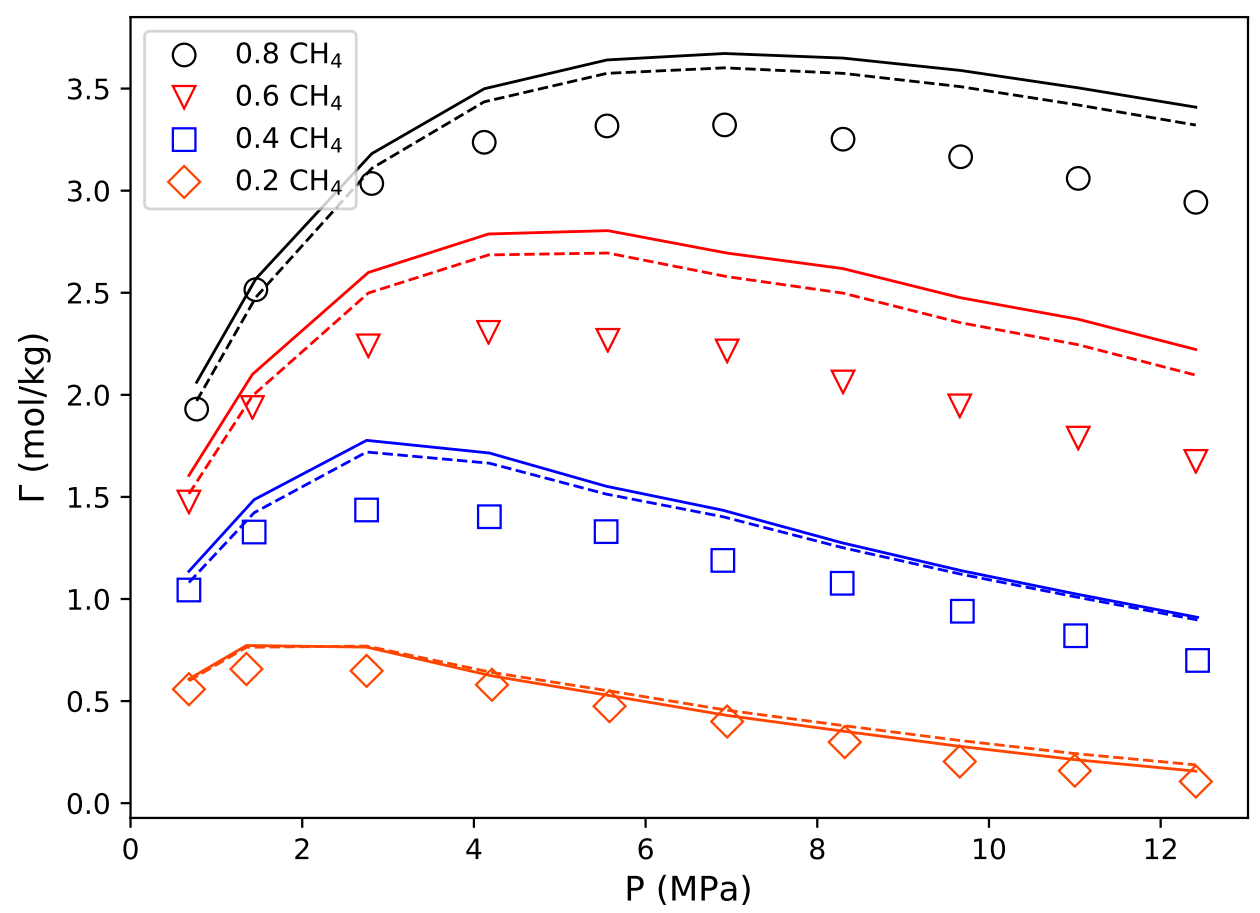

(a)

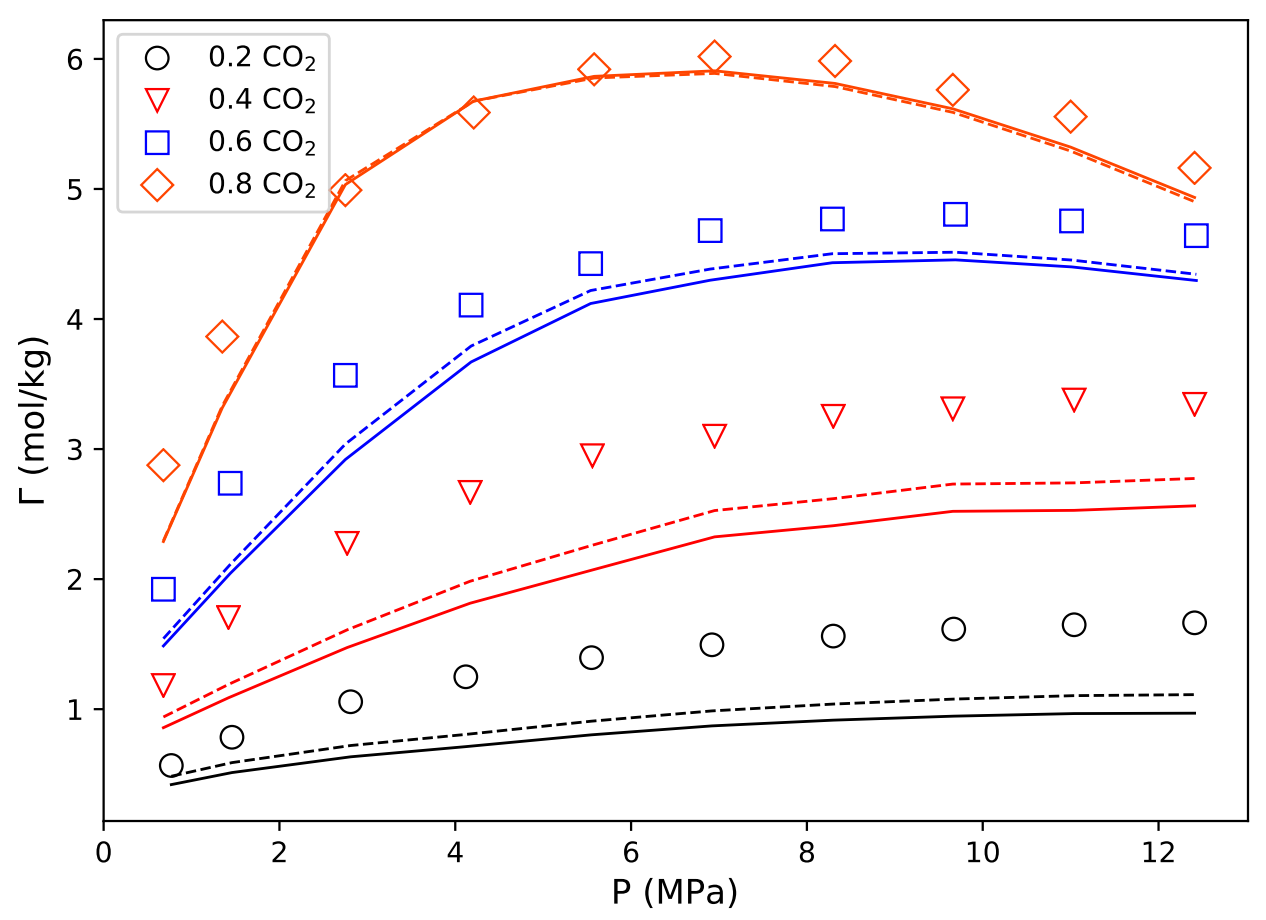

(b)

Figure 18: Prediction of adsorption of methane (a) and carbon dioxide (b) in the mixture methane-carbon dioxide $\left(k_{i j}=0.065\right)$ at $318.2 \mathrm{~K}$ on AC Calgon F400 calculated with DFTD1 and DFT-D2. Symbols are experimental data from Ref. 77. 


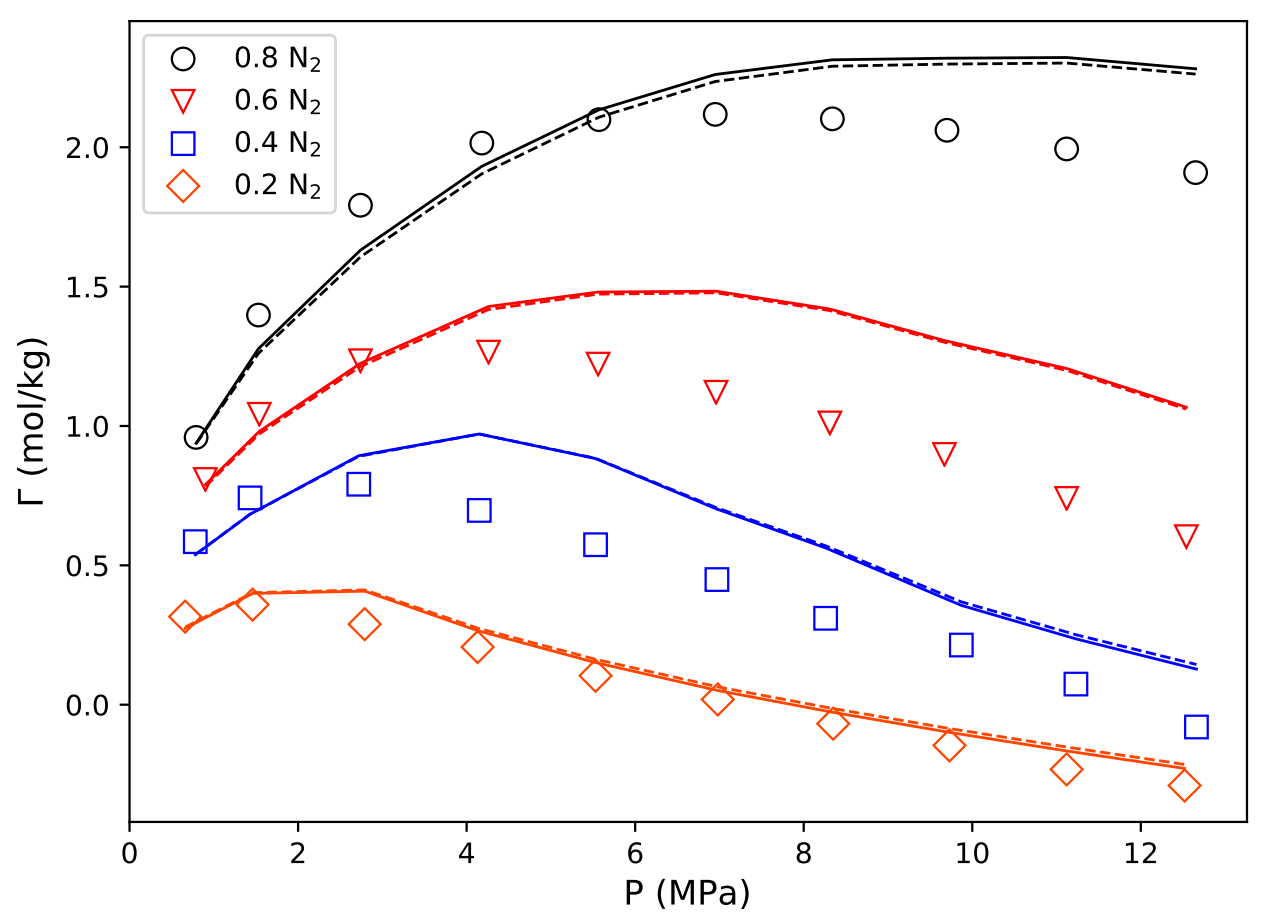

(a)

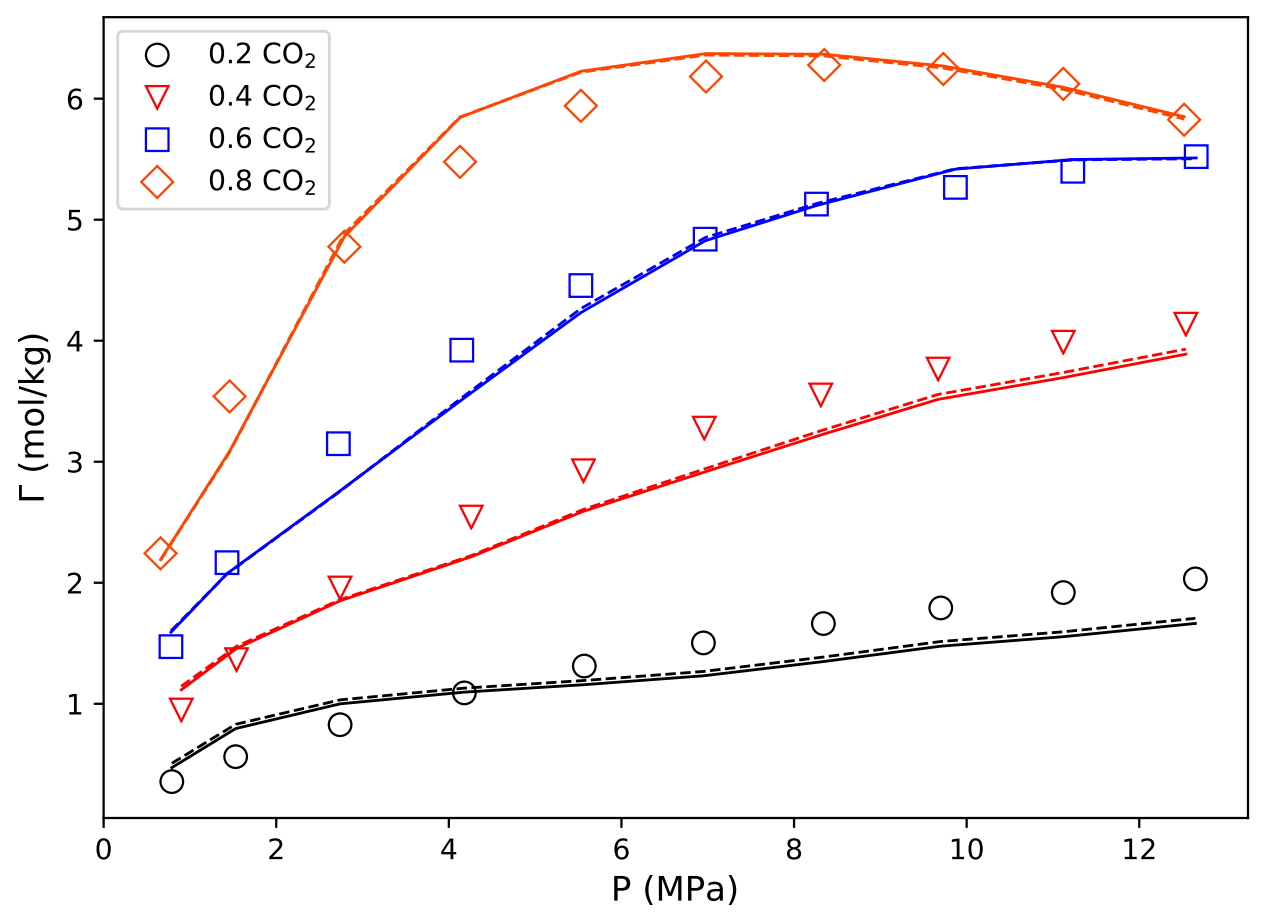

(b)

Figure 19: Prediction of adsorption of nitrogen (a) and carbon dioxide (b) in the mixture nitrogen-carbon dioxide $\left(k_{i j}=-0.0055\right)$ at $318.2 \mathrm{~K}$ on AC Calgon F400 calculated with DFT-D1 and DFT-D2. Symbols are experimental data from Ref. 77. 


\section{Conclusions}

In this work we have implemented a DFT framework for the study of interfacial tension and gas adsorption of real fluids and their mixtures. This implementation reduces to the PCSAFT equation of state in the bulk limit, and allows the use of the bulk molecular parameters of the original equation of state. To model the interactions between different compounds, the binary interaction parameters fitted to vapor-liquid equilibrium data can be used. We tested three dispersion functionals from literature with WDA that use different width of the interaction energy defined by a global parameter $\lambda$.

We proved that this approach is able to calculate the interfacial tension of pure compounds and predict the interfacial tension of their mixtures with good agreement to experimental data. In addition, DFT shows similar or higher performance than molecular dynamics simulations in the calculation of interfacial tension. It also allows the study of density profiles of the vapor-liquid interface that gives valuable insight in the structure of the fluid close to the interface at a lower cost. DFT-D2 and DFT-D3 showed a slightly better performance for the calculation of interfacial tension of mixtures for small and nearly spherical molecules, and these two 'local' approaches can be considered equivalent.

Moreover, we have shown that this implementation can be extended to the study of gas adsorption in slit-like pores. With a fairly simple framework our implementation is able to predict the effect of temperature on adsorption of pure components and also be able to predict adsorption of mixtures. Both DFT-D1 and DFT-D2 give identical results as the differences between them are compensated in the fitting procedure. It is important to note that all results of adsorption were obtained by assuming an average pore size. However, porous materials have pore size distributions that greatly affect the phase behavior and gas adsorption. Taken into account a pore size distribution may improve the accuracy of our implementation. 


\section{Acknowledgement}

The authors thank the Department of Chemical and Biochemical Engineering of the Technical University of Denmark (DTU) for funding this Ph.D. project. In addition, we also thank the support given by the SPRINT Project from the Danish Hydrocarbon Research and Technology Centre (DHRTC) at DTU.

\section{References}

(1) Kontogeorgis, G. M.; Kiil, S. Introduction to Applied Colloid and Surface Chemistry; John Wiley \& Sons, Ltd., 2016; p 367.

(2) Wu, J. Density functional theory for chemical engineering: From capillarity to soft materials. AIChE J. 2006, 52, 1169-1193.

(3) Hohenberg, P.; Kohn, W. Inhomogeneous electron gas. Phys. Rev. B 1964, 136, 864871.

(4) Ebner, C.; Saam, W. F.; Stroud, D. Density-functional theory of simple classical fluids. I. Surfaces. Phys. Rev. A 1976, 14, 2264-2273.

(5) Evans, R. In Fundamentals of Inhomogeneous Fluids; Henderson, D., Ed.; Marcel Dekker, Inc.: New York, 1992; Chapter 3, pp 85-175.

(6) Hansen, J.-P.; McDonald, I. R. Theory of simple liquids; Elsevier Academic Press, 2006; p 416.

(7) Lowen, H. Density functional theory of inhomogeneous classical fluids: recent developments and new perspectives. J. Phys.: Condens. Matter 2002, 14, 11897-11905.

(8) Wu, J.; Li, Z. Density-Functional Theory for Complex Fluids. Annu. Rev. Phys. Chem. 2007, 58, 85-112. 
(9) Emborsky, C. P.; Feng, Z.; Cox, K. R.; Chapman, W. G. Recent advances in classical density functional theory for associating and polyatomic molecules. Fluid Phase Equilib. 2011, 306, 15-30.

(10) Tarazona, P.; Evans, R. A simple density functional theory for inhomogeneous liquids. Wetting by gas at solid-liquid interface. Mol. Phys. 1984, 52, 847-857.

(11) Carnahan, N. F.; Starling, K. E. Equation of State for Nonattracting Rigid Spheres. J. Chem. Phys. 1969, 51, 635-636.

(12) Curtin, W. A.; Ashcroft, N. W. Weighted-density-functional theory of inhomogeneous liquids and the freezing transition. Phys. Rev. A 1985, 32, 2909-2919.

(13) Tarazona, P.; Cuesta, J. A.; Martínez-Ratón, Y. Density Functional Theories of Hard Particle Systems. Lect. Notes Phys. 2008, 753, 247-341.

(14) Wertheim, M. S. Fluids with highly directional attractive forces. I. Statistical Thermodynamics. J. Stat. Phys. 1984, 35, 19-34.

(15) Wertheim, M. S. Fluids with highly directional attractive forces. II. Thermodynamic perturbation theory and integral equations. J. Stat. Phys. 1984, 35, 35-47.

(16) Wertheim, M. S. Fluids with Highly Directional Attractive Forces. III. Equilibrium Polymerization. J. Stat. Phys. 1986, 42, 459-476.

(17) Wertheim, M. S. Fluids with Highly Directional Attractive Forces. IV. Equilibrium Polymerization. J. Stat. Phys. 1986, 42, 477-492.

(18) Zmpitas, W.; Gross, J. Detailed pedagogical review and analysis of Wertheim's thermodynamic perturbation theory. Fluid Phase Equilib. 2016, 428, 121-152.

(19) Jackson, G.; Chapman, W. G.; Gubbins, K. E. Phase equilibria of associating fluids: spherical and chain molecules. Int. J. Thermophys. 1988, 65, 1-31. 
(20) Chapman, W. G.; Jackson, G.; Gubbins, K. E. Phase equilibria of associating fluids: chain molecules with multiple bonding sites. Mol. Phys. 1988, 65, 1057-1079.

(21) Chapman, W. G.; Gubbins, K. E.; Jackson, G.; Radosz, M. New reference equation of state for associating liquids. Ind. Eng. Chem. Res. 1990, 29, 1709-1721.

(22) Gloor, G. J.; Jackson, G.; Blas, F. J.; Martín Del Río, E.; de Miguel, E. An accurate density functional theory for the vapor-liquid interface of associating chain molecules based on the statistical associating fluid theory for potentials of variable range. J. Chem. Phys. 2004, 121, 12740-12759.

(23) Gloor, G. J.; Jackson, G.; Blas, F. J.; Martín Del Río, E.; De Miguel, E. Prediction of the vapor-liquid interfacial tension of nonassociating and associating fluids with the SAFT-VR density functional theory. J. Phys. Chem. C 2007, 111, 15513-15522.

(24) Malheiro, C.; Mendiboure, B.; Plantier, F.; Blas, F. J.; Miqueu, C. Density functional theory for the description of spherical non-associating monomers in confined media using the SAFT-VR equation of state and weighted density approximations. J. Chem. Phys. 2014, $140,134707$.

(25) Tripathi, S.; Chapman, W. G. Microstructure of inhomogeneous polyatomic mixtures from a density functional formalism for atomic mixtures. J. Chem. Phys. 2005, 122, $1-11$.

(26) Dominik, A.; Tripathi, S.; Chapman, W. G. Bulk and interfacial properties of polymers from interfacial SAFT density functional theory. Ind. Eng. Chem. Res. 2006, 45, 67856792.

(27) Jain, S.; Dominik, A.; Chapman, W. G. Modified interfacial statistical associating fluid theory: A perturbation density functional theory for inhomogeneous complex fluids. $J$. Chem. Phys. 2007, 127, 244904. 
(28) Bymaster, A.; Chapman, W. G. An iSAFT density functional theory for associating polyatomic molecules. J. Phys. Chem. B 2010, 114, 12298-12307.

(29) Gross, J. A density functional theory for vapor-liquid interfaces using the PCP-SAFT equation of state. J. Chem. Phys. 2009, 131, 1-12.

(30) Shen, G.; Ji, X.; Lu, X. A hybrid perturbed-chain SAFT density functional theory for representing fluid behavior in nanopores. J. Chem. Phys. 2013, 138, 1-8.

(31) Shen, G.; Ji, X.; Öberg, S.; Lu, X. A hybrid perturbed-chain SAFT density functional theory for representing fluid behavior in nanopores: Mixtures. J. Chem. Phys. 2013, $139,1-9$.

(32) Shen, G.; Lu, X.; Ji, X. Modeling of molecular gas adsorption isotherms on porous materials with hybrid PC-SAFT-DFT. Fluid Phase Equilib. 2014, 382, 116-126.

(33) Klink, C.; Gross, J. A density functional theory for vapor-liquid interfaces of mixtures using the perturbed-chain polar statistical associating fluid theory equation of state. Ind. Eng. Chem. Res. 2014, 53, 6169-6178.

(34) Klink, C.; Plankova, B.; Gross, J. Density functional theory for liquid-liquid Interfaces of mixtures using the perturbed-chain polar statistical associating fluid theory equation of state. Ind. Eng. Chem. Res. 2015, 54, 4633-4642.

(35) Mairhofer, J.; Gross, J. Numerical aspects of classical density functional theory for one-dimensional vapor-liquid interfaces. Fluid Phase Equilib. 2017, 444, 1-12.

(36) Sauer, E.; Gross, J. Classical Density Functional Theory for Liquid-Fluid Interfaces and Confined Systems: A Functional for the Perturbed-Chain Polar Statistical Associating Fluid Theory Equation of State. Ind. Eng. Chem. Res. 2017, 56, 4119-4135.

(37) Wang, L.; Yang, J.; Mendenhall, J. D.; Cristancho, D. E.; Dowdle, J. R. An interfacial 
statistical associating fl uid theory (iSAFT) approach for surface/interfacial tension predictions. Fluid Phase Equilib. 2018, 476, 193-201.

(38) Lymperiadis, A.; Adjiman, C. S.; Galindo, A.; Jackson, G. A group contribution method for associating chain molecules based on the statistical associating fluid theory (SAFT-

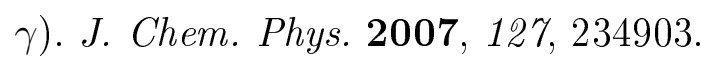

(39) Ghobadi, A. F.; Elliott, J. R. Adapting SAFT- $\gamma$ perturbation theory to site-based molecular dynamics simulation. I. Homogeneous fluids. J. Chem. Phys. 2013, 139, 234104 .

(40) Ghobadi, A. F.; Elliott, J. R. Adapting SAFT- $\gamma$ perturbation theory to site-based molecular dynamics simulation. II. Confined fluids and vapor-liquid interfaces. J. Chem. Phys. 2014, 141, 024708.

(41) Yu, Y. X.; Wu, J. Structures of hard-sphere fluids from a modified fundamental-measure theory. J. Chem. Phys. 2002, 117, 10156-10164.

(42) Yu, Y. X.; Wu, J. Density functional theory for inhomogeneous mixtures of polymeric fluids. J. Chem. Phys. 2002, 117, 2368-2376.

(43) Yu, Y. X.; Wu, J. A fundamental-measure theory for inhomogeneous associating fluids. J. Chem. Phys. 2002, 116, 7094-7103.

(44) Fu, D.; Wu, J. A self-consistent approach for modelling the interfacial properties and phase diagrams of Yukawa, Lennard-Jones and square-well fluids. Mol. Phys. 2004, 102, 1479-1488.

(45) Fu, D.; Wu, J. Vapor - Liquid Equilibria and Interfacial Tensions of Associating Fluids within a Density Functional Theory. Ind. Eng. Chem. Res. 2005, 44, 1120-1128.

(46) Rosenfeld, Y. Free-Energy Model for the Inhomogeneous Hard-Sphere Fluid Mixture and Density-Functional Theory of Freezing. Phys. Rev. Lett. 1989, 63, 980-983. 
(47) Roth, R.; Evans, R.; Lang, A.; Kahl, G. Fundamental measure theory for hard-sphere mixtures revisited: the White Bear version. J. Phys.: Condens. Matter 2002, 14, 12063.

(48) McQuarrie, D. A. Statistical Mechanics; University Science Books: Sausalito, 2000; p 641.

(49) Bymaster, A.; Dominik, A.; Chapman, W. G. Hydration structure and interfacial properties of water near a hydrophobic solute from a fundamental measure density functional theory. J. Phys. Chem. C 2007, 111, 15823-15831.

(50) Fu, J.; Tian, Y.; Wu, J. Classical Density Functional Theory for Methane Adsorption in Metal-Organic Framework Materials. AIChE J. 2015, 61, 3012-3021.

(51) Ye, Z.; Cai, J.; Liu, H.; Hu, Y. Density and chain conformation profiles of squarewell chains confined in a slit by density-functional theory. J. Chem. Phys. 2005, 123, 194902.

(52) Cahn, J. W.; Hilliard, J. E. Free energy of a nonuniform system. I. Interfacial free energy. J. Chem. Phys. 1958, 28, 258-267.

(53) Liang, X.; Michelsen, M. L.; Kontogeorgis, G. M. Pitfalls of using the geometric-mean combining rule in the density gradient theory. Fluid Phase Equilib. 2016, 415, 75-83.

(54) Liang, X.; Michelsen, M. L. General approach for solving the density gradient theory in the interfacial tension calculations. Fluid Phase Equilib. 2017, 451, 79-90.

(55) Mairhofer, J.; Gross, J. Modeling of interfacial properties of multicomponent systems using density gradient theory and PCP-SAFT. Fluid Phase Equilib. 2017, 439, 31-42.

(56) Shapiro, A. A.; Stenby, E. H. Potential theory of multicomponent adsorption. J. Colloid Interface Sci. 1998, 201, 146-157. 
(57) Monsalvo, M. A.; Shapiro, A. A. Study of high-pressure adsorption from supercritical fluids by the potential theory. Fluid Phase Equilib. 2009, 283, 56-64.

(58) Bartholdy, S.; Bjørner, M. G.; Solbraa, E.; Shapiro, A.; Kontogeorgis, G. M. Capabilities and limitations of predictive engineering theories for multicomponent adsorption. Ind. Eng. Chem. Res. 2013, 52, 11552-11563.

(59) Gross, J.; Sadowski, G. Perturbed-Chain SAFT: An Equation of State Based on a Perturbation Theory for Chain Molecules. Ind. Eng. Chem. Res. 2001, 40, 1244-1260.

(60) Boublik, T. Hard-Sphere Equation of State. J. Chem. Phys. 1970, 53, 471-472.

(61) Mansoori, G. A.; Carnahan, N. F.; Starling, K. E.; Leland, T. W. Equilibrium Thermodynamic Properties of the Mixture of Hard Spheres. J. Chem. Phys. 1971, 54, $1523-1525$.

(62) Roth, R. Fundamental measure theory for hard-sphere mixtures: a review. J. Phys.: Condens. Matter 2010, 22, 063102-18.

(63) Steele, W. A. The physical interaction of gases with crystalline solids I. Gas-solid energies and properties of isolated adsorbed atoms. Surf. Sci. 1973, 36, 317-352.

(64) P.J. Linstrom,; Mallard, W. NIST Chemistry WebBook, NIST Standard Reference Database Number 69, 20899th ed.; National Institute of Standards and Technology: Gaithersburg MD, 2018.

(65) Braun, S.; Imre, A. R.; Kraska, T. Stability limits of n-nonane calculated from molecular dynamics interface simulations. J. Chem. Phys. 2013, 138, 244710.

(66) Müller, E. A.; Mejía, A. Interfacial properties of selected binary mixtures containing n-alkanes. Fluid Phase Equilib. 2009, 282, 68-81.

(67) Sprow, F. B.; Prausnitz, J. M. Surface Tensions of Simple Liquid Mixtures. Transactions of the Faraday Society 1966, 62, 1105-1111. 
(68) Reamer, H. H.; Olds, R. H.; Sage, B. H.; Lacey, W. N. Phase Equilibria in Hydrocarbon Systems. Methane-Decane System. Ind. Eng. Chem. Res. 19342, 34, 1526-1531.

(69) Weinaug, C. F.; Katz, D. L. Surface Tensions of Methane- Propane Mixtures. Ind. Eng. Chem. Res. 1943, 35, 239-246.

(70) Amin, R.; Smith, T. N. Interfacial tension and spreading coefficient under reservoir conditions. Fluid Phase Equilib. 1998, 142, 231-241.

(71) More, J. J. In Numerical Analysis. Lecture Notes in Mathematics; G.A., W., Ed.; Springer: Berlin, Heidelberg, 1978; Vol. 630; pp 105-116.

(72) Li, M.; Gu, A.-z.; Lu, X.-S.; Wang, R.-s. Supercritical Methane Adsorption Equilibrium Data on Activated Carbon with Prediction by the Adsorption Potential Theory. J. Chem. Eng. Data 2004, 49, 73-76.

(73) Zhou, L.; Zhou, Y.; Li, M.; Chen, P.; Wang, Y. Experimental and Modeling Study of the Adsorption of Supercritical Methane on a High Surface Activated Carbon. Langmuir 2000, 16, 5955-5959.

(74) Solms, N. V.; Michelsen, M. L.; Kontogeorgis, G. M. Computational and Physical Performance of a Modified PC-SAFT Equation of State for Highly Asymmetric and Associating Mixtures. Ind. Eng. Chem. Res. 2003, 42, 1098-1105.

(75) Qiao, S.; Wang, K.; Hu, X. Study of Binary Adsorption Equilibrium of Hydrocarbons in Activated Carbon Using Micropore Size Distribution. Langmuir 2000, 16, 5130-5136.

(76) Dreisbach, F.; Staudt, R.; Keller, J. U. High pressure adsorption data of methane, nitrogen, carbon dioxide and their binary and ternary mixtures on activated carbon. Adsorption 1999, 5, 215-227.

(77) Sudibandriyo, M.; Pan, Z.; Fitzgerald, J. E.; Robinson, R. L.; Gasem, K. A. Adsorption 
of methane, nitrogen, carbon dioxide, and their binary mixtures on dry activated carbon at 318.2 K and pressures up to 13.6 MPa. Langmuir 2003, 19, 5323-5331.

(78) Justo-García, D. N.; García-Sánchez, F.; Díaz-Ramírez, N. L.; Romero-Martínez, A. Calculation of critical points for multicomponent mixtures containing hydrocarbon and nonhydrocarbon components with the PC-SAFT equation of state. Fluid Phase Equilib. 2008, 265, 192-204.

(79) Lasala, S.; Chiesa, P.; Privat, R.; Jaubert, J. N. VLE properties of CO2- Based binary systems containing N2, O2and Ar: Experimental measurements and modelling results with advanced cubic equations of state. Fluid Phase Equilib. 2016, 428, 18-31.

(80) Westman, S. F.; Stang, H. J.; Løvseth, S. W.; Austegard, A.; Snustad, I.; Størset, S. Ø.; Ertesvåg, I. S. Vapor-liquid equilibrium data for the carbon dioxide and nitrogen $(\mathrm{CO} 2+\mathrm{N} 2)$ system at the temperatures 223, 270, 298 and $303 \mathrm{~K}$ and pressures up to 18 MPa. Fluid Phase Equilib. 2016, 409, 207-241.

(81) Li, Z.; Jin, Z.; Firoozabadi, A. Phase Behavior and Adsorption of Pure Substances and Mixtures and Characterization in Nanopore Structures by Density Functional Theory. SPE J. 2014, 19, 1096-1109.

(82) Peng, D. Y.; Robinson, D. B. A New Two-Constant Equation of State. Ind. Eng. Chem. Fundam. 1976, 15, 59-64.

(83) Liu, J.; Wang, L.; Xi, S.; Asthagiri, D.; Chapman, W. G. Adsorption and Phase Behavior of Pure/Mixed Alkanes in Nanoslit Graphite Pores: An iSAFT Application. Langmuir 2017, 33, 11189-11202. 


\section{Graphical TOC Entry}

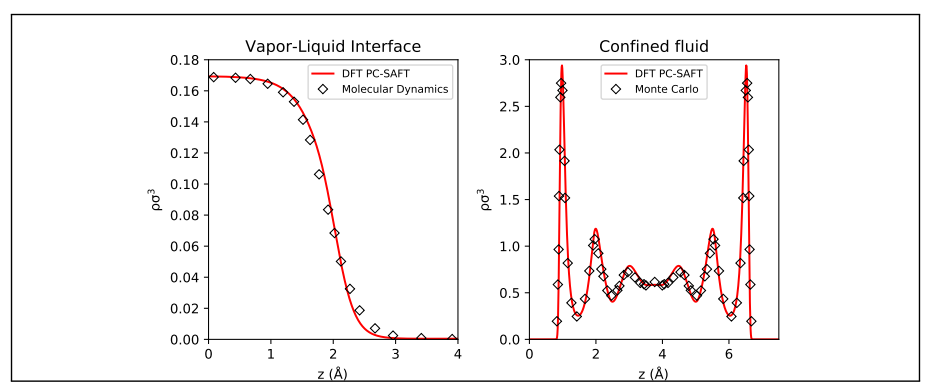

